\title{
Symmetries and the Antibracket
}

\author{
Jorge AlFARO \\ Fac. de Fisica \\ Universidad Catolica de Chile \\ Casilla 306, Santiago 22, Chile \\ Poul H. DAmgaArD* \\ Institute of Theoretical Physics \\ Uppsala University, Box 803 \\ S-751 08 Uppsala, Sweden
}

August 9, 2021

\begin{abstract}
Requiring that a Lagrangian path integral leads to certain identities (Ward identities in a broad sense) can be formulated in a general BRST language, if necessary by the use of collective fields. The condition of BRST symmetry can then be expressed with the help of the antibracket, and suitable generalizations thereof. In particular, a new Grassmann-odd bracket, which reduces to the conventional antibracket in a special limit, naturally appears. We illustrate the formalism with various examples.
\end{abstract}

UUITP-10/95

hep-th/9505156

\footnotetext{
* On leave of absence from the Niels Bohr Institute, Blegdamsvej 17, DK-2100 Copenhagen, Denmark.
} 


\section{Introduction}

In Lagrangian quantum field theory one normally starts with an action depending on a set of classical fields, and then proceeds to quantize the theory, often through the introduction of additional fields (ghosts, ghosts-for-ghosts, auxiliary fields, etc.). But the development can also proceed in the inverse order. One may wish to have imposed, already at the quantum level, a number of identities among Green functions, and is then seeking a quantum action which, together with a specified functional measure, will imply these identities. As examples of this kind, one can think of chiral lagrangians (which lead to the correct chiral Ward identities, and the associated current algebra), or quantized Yang-Mills theory (which ensures the relations generically known as Ward identities).

Gauge theories of the most general kinds can all be quantized in Lagrangian form using the remarkable framework of Batalin and Vilkovisky [1]. What is the underlying principle behind? It turns out that one can view it as the BRST principle which imposes the most general identities of any quantum field theory, the Schwinger-Dyson equations [2]. Schwinger-Dyson equations are normally not associated with any BRST symmetry, but such a connection can be established by the help of collective fields [3]. For a relation between the Batalin-Vilkovisky "quantum BRST operator" $\sigma$ and Schwinger-Dyson equations, see also ref. [4]. Imposing that the Lagrangian path integral be symmetric with respect to the Schwinger-Dyson BRST operator [3] leads directly to what is known as the Batalin-Vilkovisky (quantum) Master Equation [2]. This holds in the most general case, including that of open gauge algebras.

A crucial ingredient in the Batalin-Vilkovisky scheme is the so-called antibracket, an odd Poisson-like bracket $(\cdot, \cdot)$ defined by

$$
(F, G)=\frac{\delta^{r} F}{\delta \phi^{A}} \frac{\delta^{l} G}{\delta \phi_{A}^{*}}-\frac{\delta^{r} F}{\delta \phi_{A}^{*}} \frac{\delta^{l} G}{\delta \phi^{A}}
$$

for a set of fields $\phi^{A}$ and "antifields" $\phi_{A}^{*}$ that are canonically conjugate within this bracket,

$$
\left(\phi^{A}, \phi_{B}^{*}\right)=\delta_{B}^{A} ;\left(\phi^{A}, \phi^{B}\right)=\left(\phi_{A}^{*}, \phi_{B}^{*}\right)=0 .
$$

For flat functional measures, the Master Equation for the quantum action can be expressed entirely in terms of the antibracket and a quadratic nilpotent operator

$$
\Delta \equiv(-1)^{\epsilon_{A}+1} \frac{\delta^{r}}{\delta \phi^{A}} \frac{\delta^{r}}{\delta \phi_{A}^{*}}
$$

Here $\epsilon_{A}$ is the Grassmann parity of the fields $\phi^{A}$. The antifields $\phi_{A}^{*}$ have Grassmann parities $\epsilon_{A}+1$. (If one does not consider flat measures for the fields $\phi^{A}$, the appropriate object is a covariant generalization of $\Delta$ discussed in ref. [5, 6]; see also ref. [7].) Because of this difference in Grassmann parities, the antibracket does not share many properties with the usual Poisson bracket (and its fermionic analogue). In the anitbracket formalism the "momenta" are quite different from "coordinates", and this extends also to, e.g., ghost number assignments.

It is very simple to derive a Master Equation the quantum action $S_{\text {ext }}$ must satisfy for any theory with flat measure. One starts with the Schwinger-Dyson BRST symmetry [3]

$$
\delta \phi^{A}=c^{A}, \quad \delta c^{A}=0, \quad \delta \phi_{A}^{*}=-\frac{\delta^{l} S}{\delta \phi^{A}} .
$$

involving the fields $\phi^{A}$ and a conventional pair of ghosts $c^{A}$ and antighosts (the antifields of the Batalin-Vilkovisky formalism) $\phi_{A}^{*}$. Invariance of the path integral with respect to this symmetry 
means that the BRST variation of the action is cancelled by the Jacobian from the measure. This immediately implies

$$
\delta S_{\text {ext }}=\frac{1}{2}\left(S_{\text {ext }}, S_{\text {ext }}\right)+\frac{\delta^{r} S_{\text {ext }}}{\delta \phi^{A}} c^{A}=i \hbar \Delta S_{\text {ext }} .
$$

Or, in terms of $\psi \equiv \exp \left[(i / \hbar) S_{\text {ext }}\right]$,

$$
i \hbar \Delta \psi=c^{A} \frac{\delta^{r}}{\delta \phi^{A}} \psi
$$

The substitution $S_{\text {ext }}=S^{B V}-\phi_{A}^{*} c^{A}$ leads to the quantum Master Equation of the BatalinVilkovisky formalism:

$$
\frac{1}{2}\left(S^{B V}, S^{B V}\right)=i \hbar \Delta S^{B V}
$$

Some natural questions arise in this connection. If the most general requirement for any quantum field theory - that correct Schwinger-Dyson equations are reproduced by functional averaging of Green functions - leads to the most general BRST quantization prescription, what happens if we impose more stringent conditions? Can it be phrased in BRST language, and, if so, will the antibracket still play an important rôle? Can we impose different Master Equations to be satisfied by new classes of actions in the same manner as the Batalin-Vilkovisky Master Equation follows from demanding Schwinger-Dyson BRST symmetry?

These questions will be answered as we proceed. One of the main lessons is that the conventional antibracket formalism, being rooted in the a priori assumption of a one-to-one matching between fields and antifields, is a very special case. Figuratively speaking, by having one antifield for each field, one has completely fixed the quantum dynamics, modulo boundary conditions, of the theory. Technically, this manifests itself in the fact that all Schwinger-Dyson equations are reproduced by the BRST operator. If the number of fields does not match that of the antifields (a misleading name in this context; they are then just a particular set of ordinary antighosts of a certain BRST symmetry one wishes to impose), the quantum dynamics is not uniquely specified by the associated Master Equation. In such a case there is no natural symplectic structure in the formalism, and there is not an equal number of "coordinates" and "momenta" with which to define a canonical bracket. We emphasize that these generalized settings nevertheless are as valid as those based on the full set of Schwinger-Dyson equations. They still define classes of theories with certain specified BRST symmetries.

It is thus important to realize that the canonical structure and the antibracket itself is not required to define classes of quantum actions. The antibracket appears in the ultimate case, where the quantum theory is completely specified by the associated BRST symmetry. In all other cases some of the "momenta" $\phi^{*}$ are missing from the formalism, which is only defined on a truncated phase space. The nature of the pertinent smaller space of fields and antighosts $\phi^{*}$ can be derived from the same collective field formalism that is used to derive the Batalin-Vilkovisky scheme, and cannot be obtained by any naive truncations of the set of antighosts. The associated generalizations thus cannot be inferred without this knowledge on how to derive the Batalin-Vilkovisky formalism from a more fundamental principle [2]. Whether the existence of suitable generalizations of the Batalin-Vilkovisky Lagrangian formalism can be of use in new contexts remains to be explored (and in particular, see the comments below). We shall here restrict ourselves to deriving the main principles, and to show how known results can be understood in this new light.

What could be a motivation for trying to define classes of field theories on the basis of an underlying Master Equation for the quantum action? In the conventional antibracket formalism the answer is that it provides a systematic approach to BRST gauge fixing. All of the physical dynamics resides in the classical action and in the functional measure of the classical fields. In 
the Batalin-Vilkovisky formalism, one finds an extended action which leads to the same SchwingerDyson equations that one formally derives in the original theory, before gauge fixing. The advantage of this extended action is that it immediately can be brought in a form where the gauge has been fixed correctly, and where the path integral thus takes on a less formal significance. Similarly, even if we can find non-trivial solutions to Master Equations that lead to a more restricted set of Ward Identities, such generalizations mainly have applications at the level of gauge fixing. The classical dynamics will always be used as a boundary condition, and in this sense the Master Equations do not define for us broader classes of classical actions that will lead to the same quantum dynamics. However, and not surprisingly, the Master Equations will tell us which are the local internal symmetries of the classical action that are compatible with the required set of Ward Identities. Only in this restricted meaning does the new Master Equations stipulate conditions to be imposed on the classical actions.

The paper is organized as follows. In the next section we consider the derivation of a covariant formulation of the usual Batalin-Vilkovisky formalism, using the same procedure as in ref. [2]. (The only new ingredient is the presence of a non-trivial scalar measure density $\rho(\phi)$ in the functional integral). We show how to derive a corresponding Schwinger-Dyson BRST symmetry, and focus on the non-Abelian version of this formulation. Section 3 is devoted to most general setting possible: The case of field transformations that leave neither measure nor action invariant, and in section 4 we discuss a new Grassmann-odd bracket structure that naturally emerges in this context. Section 5 contains our conclusions. In two appendices we illustrate the BRST technique by deriving the analogue of a Batalin-Vilkovisky formalism for theories defined in terms of group variables of a given Lie group.

\section{Theories with non-trivial measures}

As a first illustration of the generality of the BRST approach to implementing chosen identities in the path integral, we shall here consider the result of imposing symmetries of a given functional measure in the form of BRST invariance. A brief collection of the main results of this section have been reported in ref. [6], but no details of the derivation were given there.

Consider a classical action $S_{c l}$ of classical fields $\phi^{A}$. Suppose we wish to quantize this theory in the path integral framework by integrating over a certain functional measure $[d \phi] \rho(\phi)$. Naively, the result would be a partition function of the form

$$
\mathcal{Z}=\int[d \phi] \rho(\phi) e^{\frac{i}{\hbar} S_{c l}[\phi]},
$$

but we know that this prescription often, and in particular if we have internal gauge symmetries, is inadequate. Obtaining correct Schwinger-Dyson equations can again be used as the principle to enlarge the path integral in a proper way. In the case of a flat functional measure, the SchwingerDyson equations were obtained by exploiting the symmetry of the measure under local translations, $\phi^{A}(x) \rightarrow \phi^{A}(x)+\varepsilon^{A}(x)$. Despite the extra factor of $\rho(\phi)$ in the measure (8), we can still make use of the invariance of just $[d \phi]$ in this case. This leads, for an arbitrary functional $F$, to one particular form of the Schwinger-Dyson equations:

$$
\left\langle(-1)^{\epsilon_{M}} \Gamma_{A M}^{M}(\phi) F(\phi)+\left(\frac{i}{\hbar}\right) \frac{\delta^{l} S}{\delta \phi^{A}} F(\phi)+\frac{\delta^{l} F}{\delta \phi^{A}}\right\rangle=0
$$


with

$$
(-1)^{\epsilon_{M}} \Gamma_{A M}^{M} \equiv \rho^{-1} \frac{\delta^{l} \rho}{\delta \phi^{A}}
$$

A more natural definition of Schwinger-Dyson equations may appear to be based on the full measure $[d \phi] \rho(\phi)$. Whereas the interesting invariance of the part $[d \phi]$ alone is a local (flat) translation, the analogous transformation in the case of $[d \phi] \rho(\phi)$ will in general take place on a non-trivial space. Subtleties can certainly arise when this space is multiply-connected, has boundaries, etc., but we shall here restrict ourselves to the case where the group of transformations which leave this full measure invariant is continuous. We are then in general dealing with a super Lie group, whose generators shall be given below.

Denote the set of transformations that leave $[d \phi] \rho(\phi)$ invariant by $g$ :

$$
\phi^{A}(x)=g^{A}\left(\phi^{\prime}(x), a(x)\right),
$$

where $a^{i}(x)$ are local fields parametrizing the transformation. They are the analogues of the local translations in the case of a flat measure. The transformation $g$ must obviously be connected to the identity, which we take to occur at $a^{i}(x)=0$ :

$$
g^{A}\left(\phi^{\prime}(x), 0\right)=\phi^{\prime A}(x) .
$$

It can happen that also the action $S$ is invariant under the set of transformations (11). This is a very special case for which, in the absence of spontaneous symmetry breaking, classical equations of motion suffice to define the full quantum theory. Certain topological field theories fall into this class. But in general the transformation (11) will not be a symmetry of the action.

Making the transformation (11) close to the identity leads to the following relations, for an arbitrary functional $G$ :

$$
\left\langle v_{i}^{B}(\phi)\left[\frac{\delta^{l} G}{\delta \phi^{B}}+\left(\frac{i}{\hbar}\right) \frac{\delta^{l} S}{\delta \phi^{B}} G(\phi)\right]\right\rangle=0,
$$

where

$$
\left.v_{i}^{A}(\phi) \equiv \frac{\delta^{l} g^{A}}{\delta a^{i}}\right|_{a=0} .
$$

These identities appear to differ from the Schwinger-Dyson equations (9). Consider, however, the case where there are locally just as many parameters $a^{i}(x)$ of the group as there are local translations. In particular, a one-to-one relation can be established with the Grassmann parities of the parameters $a^{A}$ and the original fields $\phi^{A}$, i.e., $\epsilon\left(a^{A}\right)=\epsilon\left(\phi^{A}\right)$. Now start with the original Schwinger-Dyson equations (9). Since they hold for an arbitrary $F$, they hold in particular for the choice $F_{B}^{A}(\phi)=(-1)^{\epsilon_{B}\left(\epsilon_{A}+1\right)} v_{A}^{B}(\phi) G(\phi)$. Substituting this into (9), and summing over $B$, one finds:

$$
\left\langle v_{A}^{B}(\phi)\left[\frac{\delta^{l} G}{\delta \phi^{B}}+\left(\frac{i}{\hbar}\right) \frac{\delta^{l} S}{\delta \phi^{B}}\right]+(-1)^{\epsilon_{M}} v_{A}^{B}(\phi) \Gamma_{B M}^{M}(\phi) G(\phi)+\frac{\delta^{r} v_{A}^{B}}{\delta \phi^{B}} G(\phi)\right\rangle=0 .
$$

So if one can show that

$$
\frac{\delta^{r} v_{A}^{B}}{\delta \phi^{B}}+(-1)^{\epsilon M} v_{A}^{B}(\phi) \Gamma_{B M}^{M}(\phi)=0,
$$

then the two sets of equations will be equivalent. How could an identity such as (16) arise? Our only information is that the measure $[d \phi] \rho(\phi)$ is invariant under the transformation (11). Making the substitution (11), and expanding around the identity transformation, i.e., $\phi^{A}=\phi^{A}+a^{B} v_{B}^{A}(\phi)+\ldots$, we get

$$
(-1)^{\epsilon} \rho(\phi) \frac{\delta^{r} v_{B}^{A}}{\delta \phi^{A}}+v_{B}^{A}(\phi) \frac{\delta^{l} \rho}{\delta \phi^{A}}=0 .
$$


If $v$ is invertible, this can be written

$$
\frac{\delta^{l} \rho}{\delta \phi^{C}}+(-1)^{\epsilon_{A}} \rho(\phi)\left(v^{-1}\right)_{C}^{B} \frac{\delta^{r} v_{B}^{A}}{\delta \phi^{A}}=0 .
$$

But this is precisely the relation (16), provided we identify

$$
(-1)^{\epsilon_{A}} \Gamma_{C A}^{A}=(-1)^{\epsilon_{A}+1}\left(v^{-1}\right)_{C}^{B} \frac{\delta^{r} v_{B}^{A}}{\delta \phi^{A}}
$$

which in turn is just the condition that (16) is satisfied. So under the above conditions the two sets of Schwinger-Dyson equations (9) and (13) are equivalent.

\section{$2.1 \quad$ BRST formulations}

Next, we impose the Schwinger-Dyson equations as Ward identities of an unbroken BRST symme-

try. For theories with flat measures, this has been explained in ref. [3]. When the measure is of the form $[d \phi] \rho(\phi)$, there are several routes to a proper BRST description. The simplest is to just blindly exponentiate the measure density $\rho(\phi)$, and treat this as a one-loop quantum correction to the action. We can then obviously employ the formalism of the flat measure, provided we substitute

$$
S[\phi] \rightarrow S[\phi]-i \hbar \int d x \ln [\rho(\phi)]
$$

into eq. (4). This gives

$$
\begin{aligned}
\delta \phi^{A} & =c^{A} \\
\delta c^{A} & =0 \\
\delta \phi_{A}^{*} & =-\frac{\delta^{l} S}{\delta \phi^{A}}+i \hbar(-1)^{\epsilon_{M}} \Gamma_{A M}^{M}
\end{aligned}
$$

and the Ward identities $0=\left\langle\delta\left(\phi_{A}^{*} F[\phi]\right)\right\rangle$ are then the Schwinger-Dyson equations (9). In this way one can evidently always proceed with the quantization procedure, just as in ref. [2]. But done in this way one has clearly lost all of the geometric interpretation associated with quantizing a theory on a non-trivial field space.

An alternative BRST formulation follows the second way of deriving Schwinger-Dyson equations, as described above. One here exploits the symmetry of the full measure $[d \phi] \rho(\phi)$, and promotes this symmetry into a local gauge invariance (of both measure and action). The BRST Ward identities will then be Schwinger-Dyson equations of the form (13) 10. Some of the manipulations to be considered below are clearly going to be formal in the sense that they will ignore possible modifications due to regularization. At the two-loop level certain field transformations may also require some modifications of the relevant BRST algebra [12].

In the Abelian formulation, integrate with flat measures over the collective fields $a^{A}(x)$, the auxiliary fields $B_{A}(x)$, and the ghost-antighost pair $c^{A}(x), \phi_{A}^{*}(x)$. The functional integral is then invariant under the nilpotent BRST symmetry

$$
\begin{aligned}
\delta \phi^{\prime A} & =-\left(M^{-1}\right)_{B}^{A} \frac{\delta^{r} g^{B}}{\delta a^{C}} c^{C} \\
\delta a^{A} & =c^{A} \\
\delta c^{A} & =0 \\
\delta \phi_{A}^{*} & =B_{A} \\
\delta B_{A} & =0
\end{aligned}
$$


where

$$
M_{B}^{A}\left(\phi^{\prime}, a\right) \equiv \frac{\delta^{r} g^{A}}{\delta \phi^{\prime B}} .
$$

Gauge-fixing the collective field $a$ to zero is achieved by adding a term $-\delta\left[\phi_{A}^{*} a^{A}\right]=(-1)^{\epsilon_{A}+1} B_{A} a^{A}-$ $\phi_{A}^{*} c^{A}$ to the action $S$. After integrating out $a$ and $B$, one is left with the following transformations:

$$
\begin{aligned}
\delta \phi^{A} & =-u_{B}^{A}(\phi) c^{B} \\
\delta c^{A} & =0 \\
\delta \phi_{A}^{*} & =(-1)^{\epsilon_{A}+\epsilon_{B}} \frac{\delta^{l} S}{\delta \phi^{B}} u_{A}^{B}(\phi),
\end{aligned}
$$

where we have used eq. (23), and in which

$$
\left.u_{B}^{A}(\phi) \equiv \frac{\delta^{r} g^{A}}{\delta a^{B}}\right|_{a=0} .
$$

Let us for the moment assume that $u_{B}^{A}$ is invertible, and let us then perform a simple redefinition:

$$
C^{A}=-u_{B}^{A}(\phi) c^{B}, \Phi_{A}^{*}=-\phi_{B}^{*}\left(u^{-1}\right)_{A}^{B} .
$$

Barring anomalies associated with the ghost-antighost measure $[d c]\left[d \phi^{*}\right]$, this transformation has unit Jacobian. In terms of these new variables, the BRST transformations (24) become:

$$
\begin{aligned}
\delta \phi^{A} & =C^{A} \\
\delta C^{A} & =(-1)^{\epsilon_{A} \epsilon_{D}} \Gamma_{D C}^{A} C^{C} C^{D} \\
\delta \Phi_{A}^{*} & =(-1)^{\epsilon_{D}+1} \Gamma_{A C}^{D} C^{C} \Phi_{D}^{*}-\frac{\delta^{l} S}{\delta \phi^{A}} .
\end{aligned}
$$

We have here introduced a natural connection associated with the measure $[d \phi] \rho(\phi)$, namely

$$
\Gamma_{A C}^{D} \equiv(-1)^{\epsilon_{A}\left(\epsilon_{D}+1\right)} u_{B}^{D}(\phi) \frac{\delta^{r}\left(u^{-1}\right)_{A}^{B}}{\delta \phi^{C}}
$$

One can readily check that this definition is compatible with eq. (19). Now, since the original functional measure was invariant under the transformation (11), the same should be the case for the formulation (27). Indeed, one finds that the new measure $[d \phi][d C]\left[d \Phi^{*}\right] \rho(\phi)$ is invariant under (27) precisely if

$$
\frac{\delta^{r} \rho}{\delta \phi^{D}}-(-1)^{\epsilon_{A}+\epsilon_{D}} \rho \Gamma_{D A}^{A}=0
$$

which is just the condition that the original field measure $[d \phi] \rho(\phi)$ is invariant under (11).

What about nilpotency of the BRST transformation (27)? By construction, our BRST transformations are always nilpotent when all fields of the formalism are included. When we start to integrate out part of these fields, we will in general lose nilpotency. In the present case one can easily check that nilpotency of $\delta$ in general is lost already at the level where it acts only on the original fields: $\delta^{2} \phi^{A} \neq 0$ 円. Although there is nothing wrong with such a formalism, it makes it very difficult to use it as a basis for a quantization programme. We will therefore instead focus on an alternative formulation, described below.

\footnotetext{
${ }^{1}$ Nilpotency is preserved in the case where the group of transformations is Abelian, but this is of course a very special case.
} 


\subsection{The non-Abelian formulation}

Since the set of transformations that leave the measure invariant most often will form a nonAbelian (super) Lie group, a more natural formulation of the associated Schwinger-Dyson BRST symmetry arises from the corresponding non-Abelian local gauge symmetry introduced by the help of collective fields. We shall here give a few details related to this formulation.

First a few definitions. Since the transformations (11) form a (super) group, two consecutive transformations parametrized by local fields $a^{A}(x)$ and $b^{A}(x)$ must be expressible as a single transformation of some new parameters, let us denote them by

$$
\psi^{A}(b, a)
$$

In detail, let

$$
\phi^{A}=g^{A}\left(\phi^{\prime}, a\right) \text { and } \phi^{\prime A}=g^{A}\left(\phi^{\prime \prime}, b\right),
$$

then

$$
\phi^{A}=g^{A}\left(g\left(\phi^{\prime \prime}, b\right), a\right)=g^{A}\left(\phi^{\prime \prime}, \psi(b, a)\right) .
$$

Next, differentiate this equation on both sides with respect to $b^{A}$, set $b^{A}=0$, and use the boundary conditions

$$
\psi^{A}(0, a)=a^{A} \text { and } \phi^{\prime A}=g^{A}\left(\phi^{\prime \prime}, 0\right)=\phi^{\prime A}
$$

to get

$$
\frac{\delta^{r} g^{A}\left(\phi^{\prime}, a\right)}{\delta \phi^{\prime B}} u_{C}^{B}\left(\phi^{\prime}\right)=\frac{\delta^{r} g^{A}\left(\phi^{\prime}, a\right)}{\delta a^{B}} \nu_{C}^{B}(a)
$$

Here we have introduced

$$
\left.\nu_{B}^{A}(a) \equiv \frac{\delta^{r} \psi^{A}(b, a)}{\delta b^{B}}\right|_{b=0},
$$

which has an inverse, $\lambda$, defined by

$$
\lambda_{B}^{A} \nu_{C}^{B}=\delta_{C}^{A}
$$

Consider now

$$
\delta \phi^{A}=\frac{\delta^{r} \phi^{A}\left(\phi^{\prime}, a\right)}{\delta \phi^{B}} \delta \phi^{B}+\frac{\delta^{r} \phi^{A}\left(\phi^{\prime}, a\right)}{\delta a^{B}} \delta a^{B} .
$$

It follows from (36) that if choose

$$
\begin{aligned}
\delta \phi^{\prime A} & =\left.\frac{\delta^{r} \phi^{A}\left(\phi^{\prime}, a\right)}{\delta a^{B}}\right|_{a=0} \varepsilon^{B}=u_{B}^{A}(\phi) \varepsilon^{B} \\
\delta a^{A} & =-\nu_{B}^{A}(a) \varepsilon^{B}
\end{aligned}
$$

then the original field $\phi^{A}$ is left invariant. So this is a local gauge symmetry of the transformed action. This can also be derived in a more conventional manner by starting from the Hamiltonian formulation of the collective field formalism, and then multiplying the symmetry generators by an appropriate combination of fields [11].

So far we have only verified that the gauge transformations (37) generate a symmetry of the action. Will it be a genuine quantum-mechanical symmetry in the path integral? This clearly depends on the choice of measure for the collective fields $a^{A}$. (The measure $[d \phi] \rho(\phi)$ is, by construction, invariant under the $\phi$-transformation above). As discussed in ref. [10], the functional measure for $a^{A}$ will be invariant if we choose it to be an either left or right invariant (Haar) measure. In the following we shall consider the left invariant Haar measure. 
The gauge symmetry (37) above is the non-Abelian analogue of the Abelian symmetry (27). As in all such formulations, there may be situations where there are obstructions to such a shift between Abelian and non-Abelian formulations of the same underlying gauge symmetry. What is important for our purposes is that the non-Abelian gauge symmetry (37) incorporates the conventional Schwinger-Dyson equations as BRST Ward identities. To see this, we first have to introduce the analogous BRST symmetry, and make use of some fundamentals of (super) group theory. Consider first the BRST transformations corresponding to the gauge symmetry (37):

$$
\begin{aligned}
\delta \phi^{\prime A} & =u_{B}^{A}\left(\phi^{\prime}\right) c^{B} \\
\delta a^{A} & =-\nu_{B}^{A}(a) c^{B} .
\end{aligned}
$$

Imposing nilpotency of this BRST operator $\delta$ fixes the transformation law for the ghosts $c^{A}$. After imposing $\delta^{2} \phi^{\prime A}=0$, one finds:

$$
\delta c^{A}=(-1)^{\epsilon_{B}}\left(u^{-1}\right)_{A}^{E} \frac{\delta^{r} u_{B}^{A}}{\delta \phi^{\prime C}} u_{D}^{C} c^{D} c^{B} .
$$

This transformation of the ghosts is, as usual for non-Abelian gauge symmetries, directly related to the structure coefficients $c_{B C}^{A}$ for the (super) group. One has [16]:

$$
\frac{\delta^{r} u_{B}^{A}}{\delta \phi^{\prime C}} u_{D}^{C}-(-1)^{\epsilon_{B} \epsilon_{D}} \frac{\delta^{r} u_{D}^{A}}{\delta \phi^{\prime C}} u_{B}^{C}=-u_{C}^{A} c_{B D}^{C} .
$$

The structure "coefficients" are supernumbers with the property

$$
c_{B D}^{C}=-(-1)^{\epsilon_{B} \epsilon_{D}} c_{D B}^{C} .
$$

With the help of eq. (40), one can rewrite the BRST transformation for the ghosts as

$$
\delta c^{A}=-\frac{1}{2}(-1)^{\epsilon_{B}} c_{B D}^{E} c^{D} c^{B} .
$$

Note that

$$
U_{B D}^{E} \equiv(-1)^{\epsilon_{B}} c_{B D}^{E}
$$

has the following symmetry property:

$$
U_{B D}^{E}=(-1)^{\left(\epsilon_{B}+1\right)\left(\epsilon_{D}+1\right)} U_{D B}^{E},
$$

which precisely is what is required in order that the right hand side of eq. (40), in general, is non-vanishing for arbitrary Grassmann parity assignments of the ghosts.

It still remains to be checked whether the ghost transformation law (39) is compatible with nilpotency of the BRST charge. One finds that indeed $\delta^{2} c^{A}=0$ as a consequence of the generalized Jacobi identity (see the second reference in [16]):

$$
(-1)^{\epsilon_{B} \epsilon_{E}} c_{B F}^{A} c_{C E}^{F}+(-1)^{\epsilon_{C} \epsilon_{E}} c_{E F}^{A} c_{B C}^{F}+(-1)^{\epsilon_{B} \epsilon_{C}} c_{C F}^{A} c_{E B}^{F}=0 .
$$

The transformation (39) came from requiring $\delta^{2} \phi^{A}=0$. For consistency we ought to obtain the same condition from imposing nilpotency of $\delta$ when acting on the collective fields $a^{A}$. This is indeed the case, but it interestingly turns out to involve non-trivial identities from (super) group theory. In fact, these identities can be derived from demanding a consistent BRST formulation. Demanding $\delta^{2} a^{A}=0$ leads to

$$
\delta c^{E}=(-1)^{\epsilon_{B}+1} \lambda_{A}^{E} \frac{\delta^{r} \nu_{B}^{A}}{\delta a^{C}} \nu_{D}^{C}(a) c^{D} c^{B} .
$$


Can (46) be consistent with (39)? Imposing the (super) integrability condition

$$
\frac{\delta^{r} \delta^{r} \phi^{A}}{\delta a^{C} \delta a^{B}}=(-1)^{\epsilon_{B} \epsilon_{C}} \frac{\delta^{r} \delta^{r} \phi^{A}}{\delta a^{B} \delta a^{C}}
$$

and using the composition property

$$
\frac{\delta^{r} \phi^{A}}{\delta a^{B}}=-u_{C}^{A}(\phi) \lambda_{B}^{C}(a)
$$

we obtain

$$
u_{D}^{A}\left[\frac{\delta^{r} \lambda_{B}^{D}}{\delta a^{C}}-(-1)^{\epsilon_{B} \epsilon_{C}} \frac{\delta^{r} \lambda_{C}^{D}}{\delta a^{B}}\right]-(-1)^{\epsilon_{C}\left(\epsilon_{B}+\epsilon_{D}\right)}\left[\frac{\delta^{r} u_{D}^{A}}{\delta \phi^{E}} u_{F}^{E}-(-1)^{\epsilon_{D} \epsilon_{F}} \frac{\delta^{r} u_{F}^{A}}{\delta \phi^{E}} u_{D}^{E}\right] \lambda_{C}^{F} \lambda_{B}^{D}=0 .
$$

Combining this with eq. (40) leads to the analogue of that equation, now expressed in terms of variables derived from the parameters of the group rather than the group coordinates themselves:

$$
\frac{\delta^{r} \lambda_{C}^{G}}{\delta a^{B}}-(-1)^{\epsilon_{B} \epsilon_{C}} \frac{\delta^{r} \lambda_{B}^{G}}{\delta a^{C}}=(-1)^{\epsilon_{C} \epsilon_{D}} c_{D F}^{G} \lambda_{C}^{F} \lambda_{B}^{D}
$$

This relation, eq. (50), is precisely what is needed to show that the ghost transformation (46) is equivalent to eq. (39).

To summarize this part, we have succeeded in setting up the consistent BRST multiplet associated with the collective-field gauge symmetry (37). When supplemented with a conventional antighost $\phi_{A}^{*}$ associated with the ghost $c^{A}$, and an auxiliary field $B_{A}$, we can summarize these BRST transformations below:

$$
\begin{aligned}
\delta \phi^{\prime A} & =u_{B}^{A}\left(\phi^{\prime}\right) c^{B} \\
\delta a^{A} & =-\nu_{B}^{A}(a) c^{B} \\
\delta c^{A} & =-\frac{1}{2}(-1)^{\epsilon_{B}} c_{B C}^{A} c^{C} c^{B} \\
\delta \phi_{A}^{*} & =B_{A} \\
\delta B_{A} & =0 .
\end{aligned}
$$

As before, the trick is now to integrate out the collective fields $a^{A}$ by choosing an appropriate gauge. It turns out to be convenient to introduce an object

$$
\left.\bar{\Gamma}_{B C}^{A} \equiv \frac{\delta^{r} \nu_{B}^{A}}{\delta a^{C}}\right|_{a=0}
$$

Let us first consider some of its properties. From eq. (50) it follows that

$$
\lambda_{B}^{G} \frac{\delta^{r} \nu_{K}^{B}}{\delta a^{C}} \nu_{L}^{C}-(-1)^{\epsilon_{K} \epsilon_{L}} \lambda_{B}^{G} \frac{\delta^{r} \nu_{L}^{B}}{\delta a^{C}} \nu_{K}^{C}=c_{K L}^{G}
$$

When evaluated at $a^{A}=0$, this relation implies

$$
\bar{\Gamma}_{K L}^{G}-(-1)^{\epsilon_{K} \epsilon_{L}} \bar{\Gamma}_{L K}^{G}=c_{K L}^{G}
$$

The object $\bar{\Gamma}_{B C}^{A}$ plays a role in the non-Abelian Schwinger-Dyson BRST transformations, once the collective fields $a^{A}$ (and auxiliary fields $b_{A}$ ) have been integrated out. We first gauge-fix all collective fields $a^{A}$ to zero by adding to the action a BRST gauge-fixing term of the form

$$
-\delta\left\{\phi_{A}^{*} a^{A}\right\}=(-1)^{\epsilon_{A}+1} B_{A} a^{A}+\phi_{A}^{*} \nu_{B}^{A}(a) c^{B} .
$$


Note that the ghost-antighost term is non-trivial in this formulation.

Next, let us integrate out the fields $a^{A}$ and $b_{A}$. As discussed above, we are required to take a (super) Haar measure for $a$. We can write it as $[d a]_{E} \operatorname{sdet}[\lambda(a)]$, where the subscript $E$ denotes the flat (euclidean) measure, and "sdet" denotes the superdeterminant. This means that the analogue of the equation of motion for $a^{A}$ will contain a quantum contribution as well:

$$
\frac{\delta^{r} S}{\delta a^{M}}-i \hbar \frac{\delta^{r}}{\delta a^{M}} \ln [\operatorname{sdet}(\lambda(a))]+(-1)^{\epsilon_{M}+1} B_{M}+(-1)^{\epsilon_{M}\left(\epsilon_{B}+1\right)} \phi_{A}^{*} \frac{\delta^{r} \nu_{B}^{A}}{\delta a^{M}} c^{B}=0 .
$$

Now,

$$
\begin{aligned}
\frac{\delta^{r}}{\delta a^{M}} \ln [\operatorname{sdet}(\lambda(a))]=-\frac{\delta^{r}}{\delta a^{M}} \ln [\operatorname{sdet}(\nu(a))] & =-\operatorname{sTr}\left[\nu^{-1}(a) \frac{\delta^{r} \nu(a)}{\delta a^{M}}\right] \\
& =\sum_{A}(-1)^{\epsilon}{ }^{+1} \lambda_{B}^{A}(a) \frac{\delta^{r} \nu_{B}^{A}(a)}{\delta a^{M}}
\end{aligned}
$$

which, when evaluated at $a=0$, gives

$$
(-1)^{\epsilon_{A}+1} \bar{\Gamma}_{A M}^{A}
$$

So the "quantum mechanical equation of motion" for $B$, evaluated at $a=0$, becomes

$$
B_{M}=(-1)^{\epsilon_{M}} \frac{\delta^{r} S}{\delta \phi^{A}} u_{M}^{A}(\phi)+i \hbar(-1)^{\epsilon_{A}+\epsilon_{M}} \bar{\Gamma}_{A M}^{A}+(-1)^{\epsilon_{M} \epsilon_{B}} \phi_{A}^{*} \bar{\Gamma}_{B M}^{A} c^{B} .
$$

Using the boundary condition $g^{A}\left(\phi^{\prime}, a=0\right)=\phi^{\prime A}$, this means that the BRST transformations (51) turn into

$$
\begin{aligned}
\delta \phi^{A} & =u_{B}^{A}(\phi) c^{B} \\
\delta c^{A} & =-\frac{1}{2}(-1)^{\epsilon_{B}} c_{B C}^{A} c^{C} c^{B} \\
\delta \phi_{A}^{*} & =(-1)^{\epsilon_{A}} \frac{\delta^{r} S}{\delta \phi^{B}} u_{A}^{B}(\phi)+i \hbar(-1)^{\epsilon_{A}+\epsilon_{B}} \bar{\Gamma}_{B A}^{B}+(-1)^{\epsilon_{A} \epsilon_{B}} \phi_{M}^{*} \bar{\Gamma}_{B A}^{M} c^{B} .
\end{aligned}
$$

The crucial test of the above BRST symmetry is to see if the associated Ward identities are correct Schwinger-Dyson equations. We check it by evaluating $0=\left\langle\delta\left[\phi_{A}^{*} F(\phi)\right]\right\rangle$ at the intermediate stage where the ghost-antighost pair $c^{A}, \phi_{A}^{*}$ has been integrated out. Note that the ghost expectation values in this case have to be evaluated with respect to the extended action

$$
S_{\text {ext }}=S[\phi]+\phi_{A}^{*} c^{A},
$$

with a sign difference as compared with the Abelian formulation [2].2 After a number of cancellations, one verifies that the above Ward identities turn into

$$
\left\langle(-1)^{\epsilon_{A}\left(\epsilon_{B}+1\right)} u_{A}^{B}(\phi)\left[\frac{\delta^{l} F}{\delta \phi^{B}}+\left(\frac{i}{\hbar}\right) \frac{\delta^{l} S}{\delta \phi^{B}} F(\phi)\right]\right\rangle=0,
$$

which coincide with (13).

We can write the above BRST symmetry in a more compact manner by making the redefinitions

$$
C^{A} \equiv u_{B}^{A}(\phi) c^{B}, \Phi_{A}^{*} \equiv \phi_{B}^{*}\left(u^{-1}\right)_{A}^{B},
$$

\footnotetext{
${ }^{2}$ And in particular, we now have the ghost expectation value $\left\langle c^{A} \phi_{B}^{*}\right\rangle=+i \hbar \delta_{B}^{A}$.
} 
which, as in the Abelian formulation, is a transformation of unit Jacobian, provided there are no anomalies associated with such a ghost transformation.

In terms of these new variables,

$$
\delta \phi^{A}=C^{A}
$$

so nilpotency requires $\delta C^{A}=0$. One can check that this indeed automatically is satisfied when the ghost redefinition is as given above. Finally, we can derive the transformation law for the redefined antighost $\Phi_{A}^{*}$. Defining

$$
\tilde{\Gamma}_{A K}^{M} \equiv(-1)^{\epsilon_{A}\left(\epsilon_{M}+1\right)}\left\{u_{B}^{M}(\phi) \frac{\delta^{r}\left(u^{-1}\right)_{A}^{B}}{\delta \phi^{K}}+(-1)^{\epsilon_{A} \epsilon_{C}} u_{S}^{M}(\phi) \bar{\Gamma}_{C B}^{S}\left(u^{-1}\right)_{A}^{B}\left(u^{-1}\right)_{K}^{C}\right\},
$$

we can summarize the resulting BRST transformation for all remaining fields:

$$
\begin{aligned}
\delta \phi^{A} & =C^{A} \\
\delta C^{A} & =0 \\
\delta \Phi_{A}^{*} & =\frac{\delta^{l} S}{\delta \phi^{A}}+(-1)^{\epsilon_{M}+1} \tilde{\Gamma}_{A K}^{M} C^{K} \Phi_{M}^{*}+(i \hbar)(-1)^{\epsilon_{A}+\epsilon_{C}} \bar{\Gamma}_{C B}^{C}\left(u^{-1}\right)_{A}^{B} .
\end{aligned}
$$

These "Abelianized" transformations differ slightly from the ones of eq. (27), but their Ward identities generate the same Schwinger-Dyson equations, so they are equivalent. ${ }^{3}$

It should be obvious from our derivation, but we emphasize it again here: although the extended action itself is not invariant under the transformation (65), the remaining term is precisely cancelled by a contribution from the measure, provided the original measure density $\rho(\phi)$ is covariantly conserved with respect to the connection $\Gamma_{B C}^{A}$,

$$
\rho^{-1} \frac{\delta^{r} \rho}{\delta \phi^{A}}=(-1)^{\epsilon_{S}+\epsilon_{A}} \Gamma_{A S}^{S}
$$

The only new property of $\Gamma_{B C}^{A}$ one needs in order to demonstrate this is

$$
(-1)^{\epsilon_{S}+1} \Gamma_{S A}^{S}+(-1)^{\epsilon_{S}+\epsilon_{A}} \Gamma_{A S}^{S}=0,
$$

which indeed can be derived directly from the definition (52). So BRST symmetry of the path integral is again directly linked to the measure density being covariantly conserved.

What is the Master Equation for the action in this formulation? The goal is to generalize the solution for the extended action,

$$
S_{\text {ext }}=S[\phi]+\Phi_{A}^{*} C^{A}
$$

to a more general function of the fields $\phi$ and the antighosts $\Phi^{*}$,

$$
S_{e x t}=S^{B V}\left[\phi, \Phi^{*}\right]+\Phi_{A}^{*} C^{A},
$$

while still ensuring correct Schwinger-Dyson equations for the classical action $S[\phi]$. The naive procedure would be to simply replace $S$ by $S^{B V}$ in the transformation law for $\Phi^{*}$, and then write down the condition that the extended action $S_{\text {ext }}$ is invariant under this modified BRST transformation. This is sufficient in the case of flat measures [2], but it is incorrect in the present situation. For consistency one must demand that the Master Equation for $S^{B V}$ does not involve the ghosts $C$. This is achieved if we choose

$$
\delta \phi^{A}=C^{A}
$$

\footnotetext{
${ }^{3}$ To check this, one again perform the ghost-antighost integrals in the identity $0=\left\langle\delta\left[\Phi_{A}^{*} F(\phi)\right]\right\rangle$. After a number of cancellations, one finds that the result agrees with that based on the Abelian transformations (27).
} 


$$
\begin{aligned}
\delta C^{A}= & 0 \\
\delta \Phi_{A}^{*}= & \frac{\delta^{l} S^{B V}}{\delta \phi^{A}}+(-1)^{\epsilon_{M}+1} \tilde{\Gamma}_{A K}^{M} C^{K} \Phi_{M}^{*}+(i \hbar)(-1)^{\epsilon_{A}+\epsilon_{C}} \bar{\Gamma}_{C B}^{C}\left(u^{-1}\right)_{A}^{B} \\
& +(-1)^{\epsilon_{A} \epsilon_{M}} \frac{\delta^{r} S^{B V}}{\delta \Phi_{B}^{*}} \tilde{\Gamma}_{B A}^{M} \Phi_{M}^{*}
\end{aligned}
$$

Finding the associated Master Equation for the extended action $S_{\text {ext }}$ is then only a question of demanding that the BRST variation of this extended action is cancelled by the corresponding BRST variation of the measure. With correctly imposed boundary conditions this will still ensure that correct Schwinger-Dyson equations are obtained for all fields involved. In particular, before any fixings of internal gauge symmetries are imposed, the antighosts $\Phi^{*}$ are simply set to zero by integrating over the ghosts $C$. Since $S^{B V}\left[\phi, \Phi^{*}=0\right]=S[\phi]$, the above follows.

Note that in this connection it is absolutely crucial that the extra term added to the transformation law for $\delta \Phi_{A}^{*}$,

$$
(-1)^{\epsilon_{A} \epsilon_{M}} \frac{\delta^{r} S^{B V}}{\delta \Phi_{B}^{*}} \tilde{\Gamma}_{B A}^{M} \Phi_{M}^{*},
$$

is independent of the ghosts $C$. Otherwise this term could contribute to the above Ward identities, and not yield (formally) the correct Schwinger-Dyson equations for the original theory based on $S[\phi]$.

Reinstating the Grassmann-odd BRST transformation parameter $\mu$, and denoting genuine variations (as opposed to the previous BRST variations, which change statistics)

$$
\begin{aligned}
\bar{\delta} S_{e x t}= & \frac{\delta^{r} S^{B V}}{\delta \Phi_{A}^{*}} \frac{\delta^{l} S^{B V}}{\delta \phi^{A}} \mu+(i \hbar)(-1)^{\epsilon_{A}+\epsilon_{C}} \frac{\delta^{l} S^{B V}}{\delta \Phi_{A}^{*}} \bar{\Gamma}_{C B}^{C}\left(u^{-1}\right)_{A}^{B} \mu \\
& +(i \hbar)(-1)^{\epsilon_{A}+\epsilon_{C}} \bar{\Gamma}_{C B}^{C}\left(u^{-1}\right)_{A}^{B} \mu C^{A} .
\end{aligned}
$$

The last term was the only part present when $S^{B V}\left[\phi, \Phi^{*}\right]=S[\phi]$. It was, in that case, precisely cancelled by a similar contribution from the measure.

In the present case we have to evaluate a new super Jacobian associated with the BRST transformation. Using

$$
\tilde{\Gamma}_{A B}^{B}=\Gamma_{A B}^{B}+(-1)^{\epsilon_{A}\left(\epsilon_{B}+\epsilon_{C}+1\right)} u_{S}^{B} \bar{\Gamma}_{C D}^{S}\left(u^{-1}\right)_{A}^{D}\left(u^{-1}\right)_{B}^{C},
$$

and a few identities based on the symmetry properties of $\tilde{\Gamma}_{B C}^{A}$, one finds that the cancellation of BRST variations of the extended action and the measure requires

$$
\frac{\delta^{r} S^{B V}}{\delta \Phi_{A}^{*}} \frac{\delta^{l} S^{B V}}{\delta \phi^{A}} \mu+(i \hbar)(-1)^{\epsilon_{A}+1} \frac{\delta^{r}}{\delta \phi^{A}} \frac{\delta^{r}}{\delta \Phi_{A}^{*}} S^{B V} \mu+(i \hbar)(-1)^{\epsilon_{A}+1} \rho^{-1}\left(\frac{\delta^{r} \rho}{\delta \phi^{A}}\right)\left(\frac{\delta^{r}}{\delta \Phi_{A}^{*}} S^{B V}\right) \mu=0 .
$$

This can be expressed compactly as

$$
\frac{\delta^{r} S^{B V}}{\delta \Phi_{A}^{*}} \frac{\delta^{l} S^{B V}}{\delta \phi^{A}}=-(i \hbar) \Delta_{\rho} S^{B V}
$$

with

$$
\Delta_{\rho} \equiv(-1)^{\epsilon_{A}+1} \rho^{-1} \frac{\delta^{r}}{\delta \phi^{A}}\left(\rho \frac{\delta^{r}}{\delta \Phi_{A}^{*}}\right) .
$$

\footnotetext{
${ }^{4}$ Our conventions follow those of the Appendix in ref. [2].
} 
This $\Delta_{\rho}$ is the covariant generalization of Batalin and Vilkovisky's $\Delta$-operator [1]. Equation (74) is the generalization of the quantum Master Equations to a theory with a non-trivial measure of all fields $\phi$.

When $\rho=1$, there is a direct relation between the Schwinger-Dyson BRST operator $\delta$ and the $\Delta$-operator of Batalin and Vilkovisky [2]. In the conventional way of representing the formalism, $(i \hbar) \Delta$ is viewed as a "quantum correction" to the BRST operator defined by the antibracket [4]. We now know that this particular appearance of quantum corrections to the BRST symmetry is due to the fact that the Batalin-Vilkovisky formalism is formulated at the intermediate stage where the ghosts $C^{A}$ have been integrated out of the path integral, but their antighost partners $\Phi_{A}^{*}$ have been kept. Indeed, when the measure density $\rho$ is non-trivial, the same phenomenon takes place. Just as $\Delta$ in the Master Equation is replaced by the covariant $\Delta_{\rho}$ when the measure density $\rho$ is non-trivial, the quantum correction to the BRST symmetry now becomes proportional to $(i \hbar) \Delta_{\rho}$. The identity

$$
\int[d C] F\left(C^{B}\right) \exp \left[-\frac{i}{\hbar} \Phi_{A}^{*} C^{A}\right]=F\left(i \hbar \frac{\delta^{l}}{\delta \Phi_{B}^{*}}\right) \int[d C] \exp \left[-\frac{i}{\hbar} \Phi_{A}^{*} C^{A}\right]
$$

suffices to show this. It tells us how to correctly replace the ghosts $C^{A}$ in the BRST variations when these fields have been integrated out. The Schwinger-Dyson BRST variation of an arbitrary functional $G\left[\Phi, \Phi^{*}\right]$ can, inside the functional integral (where partial integrations are allowed), then be rewritten:

$$
\begin{aligned}
\delta G\left[\phi, \Phi^{*}\right] & \frac{\delta^{r} G}{\delta \phi^{A}} C^{A}+\frac{\delta^{r} G}{\delta \Phi_{A}^{*}}\left\{(-1)^{\epsilon_{M}+1} \Gamma_{A K}^{M} C^{K} \Phi_{M}^{*}+(-1)^{\epsilon_{A} \epsilon_{M}+1} \frac{\delta^{r} S^{B V}}{\delta \Phi_{K}^{*}} \Gamma_{K A}^{M} \Phi_{M}^{*}-\frac{\delta^{l} S^{B V}}{\delta \phi^{A}}\right\} \\
& \rightarrow \frac{\delta^{r} G}{\delta \phi^{A}} \frac{\delta^{l} S^{B V}}{\delta \Phi_{A}^{*}}-\frac{\delta^{r} G}{\delta \Phi_{A}^{*}} \frac{\delta^{l} S^{B V}}{\delta \phi^{A}}+(i \hbar)\left((-1)^{\epsilon} \frac{\delta^{r}}{\delta \phi^{A}}+(-1)^{\epsilon_{A} \epsilon_{G}+\epsilon_{M}} \Gamma_{A M}^{M}\right) \frac{\delta^{r}}{\delta \Phi_{A}^{*}} G .
\end{aligned}
$$

The arrow has indicated where a partial integration is required. Furthermore, by means of the identity (76) we have

$$
\Delta_{\rho} G=(-1)^{\epsilon_{A}+1} \rho^{-1} \frac{\delta^{r}}{\delta \phi^{A}}\left(\rho \frac{\delta^{r} G}{\delta \Phi_{A}^{*}}\right)=\left((-1)^{\epsilon_{A}+1} \frac{\delta^{r}}{\delta \phi^{A}}+(-1)^{\epsilon_{A} \epsilon_{G}+\epsilon_{M}+1} \Gamma_{A M}^{M}\right) \frac{\delta^{r}}{\delta \Phi_{A}^{*}} G .
$$

So the equivalent of the Schwinger-Dyson BRST operator at the intermediate stage where one has integrated out the ghosts $C^{A}$ is given by (in a notation similar to ref. [《]),

$$
\sigma_{\rho} \equiv\left(\cdot, S^{B V}\right)-i \hbar \Delta_{\rho}
$$

in the covariant formulation. Note that the action $S^{B V}$ is not a generator of the correct BRST symmetry within the antibracket: it picks up only the classical part of the Schwinger-Dyson equations. This holds even if the measure density $\rho$ is trivial.

As a simple check of the above manipulations, consider evaluating the Ward identity

$$
\left\langle\sigma_{\rho} \Phi_{A}^{*} F(\phi)\right\rangle=0 \text {. }
$$

in the case where there are no internal gauge symmetries, and $S^{B V}\left[\phi, \Phi^{*}\right]$ therefore can be taken to equal $S[\phi]$. It is straightforward to confirm that the result coincides with eq. (61). When there are internal gauge symmetries, it is important to choose correct boundary conditions for $S^{B V}$ in order to keep the correct Schwinger-Dyson equations as Ward identities, completely analogous to the case of trivial measure density $\rho=1$ [2]. 


\subsection{Example: a space with torsion}

A specific example may be instructive at this point. Suppose we are given a space of fields, a manifold (and let us for simplicity choose it to be entirely bosonic), and suppose that we are provided with a natural connection $\Gamma_{B C}^{A}$ on this manifold. Can we set up a suitable quantization procedure for a theory of action $S_{\text {ext }}$ defined on such a manifold? Since nothing has been assumed about the symmetry properties of the connection $\Gamma_{B C}^{A}$, we split it up into its symmetric and antisymmetric combinations (with respect to the lower indices):

$$
\Gamma_{B C}^{A}=\frac{1}{2}\left(\Gamma_{B C}^{A}+\Gamma_{C B}^{A}\right)+S_{B C} \cdot{ }^{A},
$$

where the torsion tensor $S_{\overrightarrow{B C}} \cdot A$ is defined to the antisymmetric part of the connection:

$$
S_{B C}^{A} \equiv \frac{1}{2}\left[\Gamma_{B C}^{A}-\Gamma_{C B}^{A}\right]
$$

We shall now seek the appropriate Schwinger-Dyson BRST algebra for field spaces with torsion. The only modification in comparison with the (bosonic) curved, but torsionless, case is that the connection $\Gamma_{B C}^{A}$ is no longer symmetric in the lower indices. As far as the action is concerned, BRST invariance hinges in the bosonic case crucially on this symmetry. This means that if we blindly substitute the connection (81) into the conventional Schwinger-Dyson BRST transformations (27), the action will no longer be invariant. We can cure for this by taking only a specific symmetric combination $\Lambda_{B C}^{A}$ defined by

$$
\Lambda_{B C}^{A} \equiv \frac{1}{2}\left[\Gamma_{B C}^{A}+\Gamma_{C B}^{A}\right]+\left[S_{B C}^{A \cdots}+S_{C B}^{A} \cdot \dot{ } .\right.
$$

The action is now guaranteed to be invariant under the BRST transformations

$$
\begin{aligned}
\delta \phi^{A} & =c^{A} \\
\delta c^{A} & =0 \\
\delta \phi_{A}^{*} & =\Lambda_{B A}^{C} c^{B} \phi_{C}^{*}-\frac{\delta S}{\delta \phi^{A}}
\end{aligned}
$$

but we still need to check that the functional measure is invariant as well. This is the case if

$$
\frac{\delta \rho}{\delta \phi^{A}}-\Lambda_{A B}^{B} \rho=0
$$

which, on account of eq. (83), is equivalent to

$$
\frac{\delta \rho}{\delta \phi^{A}}-\Gamma_{A B}^{B} \rho=0
$$

that is, precisely the condition that $\rho$ is covariantly conserved with respect to the connection $\Gamma_{B C}^{A}$. An invariant measure defined through this criterion will thus satisfy all requirements.

To make these considerations even more concrete, let us study explicitly a particular case: a Riemann-Cartan space defined by a metric-preserving connection $\Gamma_{B C}^{A}$, which can be written in terms of the Christoffel symbol and the so-called contortion tensor $K_{B C} \cdot A$ in the following way [8]:

$$
\Gamma_{B C}^{A}=\{\underset{B C}{A}\}-K_{B C}^{\cdot A} .
$$

The tensors $K$ and $S$ are related to each other:

$$
K_{B C} \cdot A=-S_{B C} \cdot A+S_{B} \cdot A \cdot \dot{C}-S^{A} \cdot \ddot{B C} \cdot
$$


An explicit expression for an invariant measure can be found by solving the constraint that $\rho$ be covariantly conserved with respect to $\Gamma$, eq. (86). An integrability condition is that a trace of the torsion tensor can be derived from a scalar potential, $S_{A B}^{A}=\partial \Theta / \partial \phi^{B}$, in terms of which (see, e.g., ref. [9]):

$$
\rho(\phi)=e^{2 \Theta(\phi)} \sqrt{g(\phi)} .
$$

We now add to the action the same $\phi_{A}^{*} c^{A}$-term as before. The BRST Ward identities $0=$ $\left\langle\delta\left(\phi_{A}^{*} F[\phi]\right)\right\rangle$ correspond, after integrating out the ghost-antighost pair $c^{A}, \phi_{A}^{*}$, to the SchwingerDyson equations for a theory of action $S$ and measure density $\rho(\phi)$ satisfying eq. (86). The BRST symmetry (84) is therefore the sought-for Schwinger-Dyson BRST symmetry for this case.

\section{Master Equations for other symmetries}

While Schwinger-Dyson equations are the most general identities of any quantum field theory, there are of course interesting subsets that can play very important rôles. The Ward identities of ordinary gauge symmetries, chiral Ward identities, conformal Ward identities, etc., are all examples that illustrate the importance of having exact identities which one can demand must be satisfied by a quantum theory. Thus, while Schwinger-Dyson equations can serve to define the full Lagrangian quantum theory [2], there may be less general equations that one wishes to impose in the process of quantization. Having realized how to derive the Batalin-Vilkovisky Lagrangian formalism [i] from the Schwinger-Dyson BRST symmetry [3], it is of course not a big step to generalize this to an arbitrary class of transformations. This is the subject we turn to next.

The idea is to promote an arbitrary symmetry of action or of measure (or of both) into a BRST symmetry. The original symmetry may be local or global, and it is irrelevant if it is spontaneously broken. In fact, the symmetry may even be anomalous in the sense that it is broken by quantum corrections. To make the picture complete, we can even demand that the BRST symmetry is based on transformations of the original fields that leave neither the action nor the measure, nor even the combination of the two, invariant. That this is possible, is due to the enormous freedom we have available in defining the Lagrangian path integral.

\subsection{Non-invariant measures}

Suppose we are presented with a partition function

$$
\mathcal{Z}=\int[d \phi] \rho(\phi) e^{\frac{i}{\hbar} S[\phi]}
$$

In the previous section we discussed the set of transformations (37) that left the functional measure $[d \phi] \rho(\phi)$ invariant. Here we want to be more general, and consider this theory in the light of arbitrary transformations $\phi^{A}=g^{A}\left(\phi^{\prime}, a\right)$, independently of whether they leave the measure invariant or not. What is the modification? When we perform the transformation that takes us from $\phi$-variables to $\phi^{\prime}$-variables, the measure changes due to the Jacobian:

$$
\int[d \phi] \rho(\phi) \rightarrow \int\left[d \phi^{\prime}\right] \rho\left(\phi^{\prime}, a\right) \operatorname{sdet}(M),
$$

where the matrix $M_{B}^{A}$ is as defined in eq. (23), and where $\rho\left(\phi^{\prime}, a\right)=\rho\left(\phi\left(\phi^{\prime}, a\right)\right)$. The fact that the measure changes due to the field transformation does not affect the new gauge symmetry between 
the transformed fields $\phi^{\prime}$ and and the collective fields $a$. Consider the Abelian formulation. We here integrate $a$ over the flat measure, which is invariant under the gauge transformation $\delta a^{i}=\varepsilon^{i}$. The full measure (91) for $\phi^{\prime}$ is now invariant under the gauge transformation corresponding to (22). This is obvious from its construction (the measure was originally only a function of the fields $\phi^{A}$, and these are gauge invariant by themselves), but it is far from obvious when one considers the definition (91) of the $\phi^{\prime}$-measure. It is therefore worthwhile to check the invariance. We use the fact that

$$
\delta[\operatorname{sdet}(M)]=\operatorname{sdet}(M) \operatorname{sTr}\left[M^{-1} \delta M\right] .
$$

The measure density $\rho\left(\phi^{\prime}, a\right)=\rho(\phi)$ is explicitly invariant by itself, since it is only a function of $\phi$. Finally, from the $\left[d \phi^{\prime}\right]$ part we get an extra term

$$
\mathrm{s} \operatorname{Tr}\left[\frac{\delta^{r} \delta \phi^{\prime}}{\delta \phi^{\prime}}\right]
$$

It follows from eq. (23) that

$$
\begin{aligned}
\frac{\delta^{r} \delta \phi^{\prime A}}{\delta \phi^{\prime C}} & =-\left(M^{-1}\right)_{B}^{A} \frac{\delta^{r} M_{C}^{B}}{\delta a^{i}} \delta a^{i}+(-1)^{\epsilon_{C}} \epsilon_{B} \frac{\delta^{r}\left(M^{-1}\right)_{B}^{A}}{\delta \phi^{\prime C}} M_{D}^{B} \delta \phi^{\prime D} \\
& =-\left(M^{-1}\right)_{B}^{A} \frac{\delta^{r} M_{C}^{B}}{\delta a^{i}} \delta a^{i}-\left(M^{-1}\right)_{B}^{A} \frac{\delta^{r} M_{C}^{B}}{\delta \phi^{D}} \delta \phi^{\prime D} \\
& =-\left(M^{-1}\right)_{B}^{A} \delta M_{C}^{B},
\end{aligned}
$$

which shows that the Jacobian from $\left[d \phi^{\prime}\right]$ is cancelled by the change in $\operatorname{sdet}(M)$. So the theory defined formally by

$$
\mathcal{Z}=\int[d a]\left[d \phi^{\prime}\right] \rho\left(\phi^{\prime}, a\right) \operatorname{sdet}\left[M\left(\phi^{\prime}, a\right)\right] e^{\frac{i}{\hbar} S\left[\phi^{\prime}, a\right]}
$$

has the gauge symmetry (37). The measure and the action are separately invariant. The transformations $g^{A}\left(\phi^{\prime}, a\right)$ can hence be chosen completely arbitrary; the path integral enlarged with the help of the collective fields $a$ is still defining a gauge invariant theory. We only need to include a possible Jacobian factor $\operatorname{sdet}(M)$, as shown above.

If the transformations are chosen arbitrarily, how can they contain any physical information? They can because they probe the response of the path integral to a reparametrization. If we perform functional averages, these probes of the path integral give rise to identities, in general a subset of the complete set of Schwinger-Dyson equations. This is no more surprising than the previous case of invariant measures: also here the only information concerns properties of the measure, and is completely independent of the action $S$ under consideration.

The procedure is now the same as before: when we integrate out the collective fields $a$ after having fixed an appropriate gauge, the left-over BRST symmetry will give rise to non-trivial Ward identities. Of course, these Ward identities are not new. Just as in the case of Schwinger-Dyson equations, they can be derived straightforwardly by manipulations directly at the path-integral level. However, as with Schwinger-Dyson equations, one advantage of the corresponding BRST formulation is that the identities can be imposed on the path integral by means of a non-trivial condition on the quantum action - a generalized Master Equation. One can then select the whole class of actions that will yield the same identities, for all the fields involved.

As before, we fix $a^{i}=0$ by adding to the action a term of the form $-\delta\left[\phi_{A}^{*} a^{A}\right]$. The BRST operator is the same as in eq. (22), since the gauge symmetry is unaffected by the presence of 
the extra $\operatorname{sdet}(M)$ term in the measure. Integrating out $B_{i}$ and $a^{i}$ we do, however, get a slight modification of the residual BRST transformation law:

$$
\begin{aligned}
\delta \phi^{A} & =-u_{i}^{A}(\phi) c^{i} \\
\delta c^{i} & =0 \\
\delta \phi_{i}^{*} & =(-1)^{\epsilon_{i}}\left[\frac{\delta^{r} S}{\delta \phi^{A}} u_{i}^{A}(\phi)-(i \hbar)\left\{\frac{\delta^{r} \ln \rho}{\delta \phi^{A}} u_{i}^{A}+\left.\frac{\delta^{r} \ln \operatorname{sdet}(M)}{\delta a^{i}}\right|_{a=0}\right\}\right] .
\end{aligned}
$$

In the case of a measure invariant under the substitution $\phi^{A}=g^{A}\left(\phi^{\prime}, a\right)$, the last two terms cancel each other.

We find the relevant BRST Ward identities as before by evaluating the ghost-antighost expectation value $0=\left\langle\delta\left[\phi_{i}^{*} F(\phi)\right]\right\rangle$. This gives

$$
\left\langle(-1)^{\epsilon_{i}\left(\epsilon_{A}+1\right)} u_{i}^{A}\left[\frac{\delta^{l} F}{\delta \phi^{A}}+\left(\frac{i}{\hbar}\right) \frac{\delta^{l} S}{\delta \phi^{A}} F\right]\right\rangle=\left\langle(-1)^{\epsilon_{i}+1}\left[\frac{\delta^{r} \ln \rho}{\delta \phi^{A}} u_{i}^{A}+\left.\frac{\delta^{r} \ln \operatorname{sdet}(M)}{\delta a^{i}}\right|_{a=0}\right]\right\rangle .
$$

While the identities (96) are not as general as the full set of Schwinger-Dyson equations, there are many examples in field theory where they play an important rôle. Typical cases may involve anomalous Ward identities, where the measure can be formally invariant under a group of transformations, but where this symmetry is broken by the ultraviolet regulator such as a set Pauli-Villars fields. If these fields are integrated out of the path integral, they will provide a non-invariant measure for the original variables. The right hand side of eq. (96) then provides the violation of the naive Ward identity.

As with the case of invariant measures, the present Abelian formulation suffers from the problem that the BRST charge is not nilpotent when acting on the fields $\phi$. It fortunately takes little work to see that the new measure

$$
\int[d a]_{E}\left[d \phi^{\prime}\right]_{E} \rho\left(\phi^{\prime}, a\right) \operatorname{sdet} \lambda(a) \operatorname{sdet} M\left(\phi^{\prime}, a\right)
$$

is invariant under the corresponding non-Abelian gauge transformation (37) as well. In order to compute the super Jacobian of the transformation, we first evaluate

$$
\frac{\delta^{r} \delta \phi^{\prime A}}{\delta \phi^{\prime B}}=(-1)^{\epsilon_{B}} \epsilon_{i} \frac{\delta^{r} u_{i}^{A}}{\delta \phi^{B}} \varepsilon^{i}
$$

Next, differentiating the identity (25) with respect to $\phi^{\prime}$, we find:

$$
\frac{\delta^{r} u_{j}^{D}}{\delta \phi^{\prime C}}=\left(M^{-1}\right)_{A}^{D}\left[(-1)^{\epsilon_{i} \epsilon_{C}} \frac{\delta^{r} M_{C}^{A}}{\delta a^{i}} \nu_{j}^{i}-(-1)^{\epsilon_{C}\left(\epsilon_{B}+\epsilon_{j}\right)} \frac{\delta^{r} M_{B}^{A}}{\delta \phi^{\prime C}} u_{j}^{B}\right]
$$

which means that

$$
\begin{aligned}
\frac{\delta^{r} \delta \phi^{\prime A}}{\delta \phi^{\prime B}} & =\left(M^{-1}\right)_{C}^{A}\left[\frac{\delta^{r} M_{B}^{C}}{\delta a^{j}} \nu_{i}^{j}-(-1)^{\epsilon_{B} \epsilon_{D}} \frac{\delta^{r} M_{D}^{C}}{\delta \phi^{B}} u_{i}^{D}\right] \varepsilon^{i} \\
& =\left(M^{-1}\right)_{C}^{A}\left[\frac{\delta^{r} M_{B}^{C}}{\delta a^{j}} \nu_{i}^{j}-\frac{\delta^{r} M_{B}^{C}}{\delta \phi^{\prime D}} u_{i}^{D}\right] \varepsilon^{i} \\
& =-\left(M^{-1}\right)_{C}^{A} \delta M_{B}^{C} .
\end{aligned}
$$

As in the Abelian case, the Jacobian from $\left[d \phi^{\prime}\right]$ is therefore precisely cancelled by the change in $\operatorname{sdet}(M)$ : the new measure is invariant under the non-Abelian gauge symmetry. 
At the level where all fields are kept, the corresponding non-Abelian BRST transformations obviously coincide with those of eq. (37). Differences only show up when we gauge-fix the symmetry (by, say, adding the conventional $-\delta\left\{\phi_{i}^{*} a^{i}\right\}$ term to the action) and then integrate out both the collective fields $a^{i}$ and the Nakanishi-Lautrup fields $B_{i}$. Using the same technique as in the section above, we find that only the transformation law for $\phi_{i}^{*}$ is modified:

$$
\begin{aligned}
\delta \phi_{i}^{*}=\quad & (-1)^{\epsilon_{i}} \frac{\delta^{r} S}{\delta \phi^{A}} u_{i}^{A}(\phi)+(-1)^{\epsilon_{i} \epsilon_{j}} \phi_{k}^{*} \bar{\Gamma}_{j i}^{k} c^{j} \\
& +(i \hbar)\left[(-1)^{\epsilon_{i}+\epsilon_{j}} \bar{\Gamma}_{j i}^{j}+(-1)^{\epsilon_{i}+1}\left\{\frac{\delta^{r} \ln \rho}{\delta \phi^{A}} u_{i}^{A}(\phi)+N_{i}\right\}\right],
\end{aligned}
$$

where we have defined

$$
\left.N_{i} \equiv \frac{\delta^{r} \ln \operatorname{sdet}(M)}{\delta a^{i}}\right|_{a=0}=\left.(-1)^{\epsilon} \frac{\delta^{r} M_{A}^{A}}{\delta a^{i}}\right|_{a=0}
$$

\subsection{The corresponding Master Equation}

Although the action $S_{\text {ext }}$ is not invariant under (100), we also here have the situation that its BRST variation $\delta S_{\text {ext }}$ is cancelled by a contribution from the measure. So far we have assumed that $S$ is a function of the fields $\phi^{A}$ only. We can easily generalize this to an arbitrary action $S_{\text {ext }}$ that depends on both the fields $\phi^{A}$ and, say, the antighosts $\phi_{A}^{*}$. As before, depending on the manner in which we generalize the action, we are forced to modify the BRST transformation laws as well. Since we wish to preserve nilpotency of the BRST charge $Q$ when acting on the fundamental fields $\phi^{A}$, the only possibility is to modify the transformation law for the antighosts $\phi^{*}$.

Let us again restrict ourselves to the generalization

$$
S \rightarrow S_{\text {ext }}=S^{B V}\left[\phi, \phi^{*}\right]+\phi_{i}^{*} c^{i}
$$

Using the ansatz

$$
\begin{aligned}
\delta \phi_{i}^{*}=\quad & (-1)^{\epsilon_{i}} \frac{\delta^{r} S^{B V}}{\delta \phi^{A}} u_{i}^{A}(\phi)+(-1)^{\epsilon_{i} \epsilon_{j}} \phi_{k}^{*} \bar{\Gamma}_{j i}^{k} c^{j}+M_{i} \\
& +(i \hbar)\left[(-1)^{\epsilon_{i}+\epsilon_{j}} \bar{\Gamma}_{j i}^{j}+(-1)^{\epsilon_{i}+1}\left\{\frac{\delta^{r} \ln \rho}{\delta \phi^{A}} u_{i}^{A}(\phi)+N_{i}\right\}\right],
\end{aligned}
$$

we determine the extra term $M_{i}$ from the requirement that the corresponding Master Equation for $S^{B V}$ is independent of the ghosts $c^{i}$ (since this would contradict the assumption that $S^{B V}$ depends only upon $\phi^{A}$ and $\phi_{i}^{*}$ ). As the associated Master Equation will contain both classical and quantum (proportional to $\hbar$ ) parts, the possibility of finding a consistent solution for $M_{i}$ is not at all guaranteed. At the classical level, we find that the two terms in $\bar{\delta} S_{\text {ext }}$ which involve two factors of ghosts $c$ cancel each other automatically, independently of the choice of $M_{i}$. Staying at the classical level, terms involving only one factor of $c$ cancel if

$$
M_{i}=(-1)^{\epsilon_{i}\left(\epsilon_{j}+1\right)} \frac{\delta^{r} S^{B V}}{\delta \phi_{j}^{*}} \phi_{k}^{*} \bar{\Gamma}_{i j}^{k} .
$$

\footnotetext{
${ }^{5}$ To ensure that this extended action $S_{\text {ext }}$ has zero ghost number, this of course requires the presence of fields $\phi^{A}$ with non-zero ghost number. Such ghost fields can either already be inherent in the formalism (required by the subsequent gauge fixing of internal symmetries), or they can be added by hand.
} 
This also immediately gives us the classical Master Equation:

$$
(-1)^{\epsilon_{i}} \frac{\delta^{r} S^{B V}}{\delta \phi_{i}^{*}} \frac{\delta^{r} S^{B V}}{\delta \phi^{A}} u_{i}^{A}(\phi)+\frac{1}{2}(-1)^{\epsilon_{i}\left(\epsilon_{j}+1\right)} \frac{\delta^{r} S^{B V}}{\delta \phi_{i}^{*}} \frac{\delta^{r} S^{B V}}{\delta \phi_{j}^{*}} \phi_{k}^{*} c_{i j}^{k}=0 .
$$

Note that the usual antibracket does not appear here (there are not, in general, an equal number of "coordinates" $\phi^{A}$ and "momenta" $\phi_{i}^{*}$, so this was ruled out from the beginning). And a new term, proportional to the structure coefficients of the group of transformations has emerged.

Being described purely at the classical level, the above Master Equation of course makes no reference to the functional measure, and to whether this measure is invariant under the field transformation $\phi^{A}=g^{A}\left(\phi^{\prime}, a\right)$ or not. This means that the same Master Equation should emerge even in the more conventional case described in section 2 where we considered the case of a one-to-one matching between fields $\phi^{A}$ and collective fields $a^{A}$. When the transformation matrix $u_{B}^{A}(\phi)$ was invertible, we could show that the associated classical Master Equation was nothing but the usual antibracket relation

$$
\left(S^{B V}, S^{B V}\right)=0 .
$$

On the surface, this would seem to contradict the derivation presented here. The case of a one-toone matching of degrees of freedom between $\phi^{A}$ and $a^{A}$, and an invertible $u_{B}^{A}(\phi)$, is but a special case of the above more general considerations. Fortunately, there is no contradiction. The difference between these two alternative descriptions lies, in the special case referred to in section 2 in the choice of antighosts $\phi^{*}$. When $u_{B}^{A}$ is invertible, we can define new ghosts $C^{A}$ and new antighosts $\Phi_{A}^{*}$ according to eq. (62). This does not affect the action

$$
S_{\text {ext }}=S[\phi]+\phi_{A}^{*} c^{A},
$$

which is invariant under such a substitution. But when we next generalize the extended action to include an antighost-dependence in the main part, the Master Equation for

$$
S_{\text {ext }}=S\left[\phi, \phi^{*}\right]+\phi_{A}^{*} c^{A}
$$

will of course differ from

$$
S_{\text {ext }}=S\left[\phi, \Phi^{*}\right]+\Phi_{A}^{*} C^{A}
$$

due to the implicit $\phi$-dependence in $\Phi^{*}$. In fact, the term needed to provide the same solution (in terms of the same variables) for these two equations is precisely the "commutator" term in eq. (105) above.

The fact that $M_{i}$ turns out to involve only one power of $\phi^{*}$, and none of the ghosts $c$, is crucial for the consistency of this procedure. This way the Ward identities for $S_{\text {ext }}$ will, formally, coincide with the Schwinger-Dyson equations for the original action $S[\phi]$ after integrating over the ghosts $c$ and antighosts $\phi^{*}$. This however, is not the only consistency check. Although the choice (104) guarantees the absence of terms involving the ghosts $c$ in the classical Master Equation for $S^{B V}$, nothing would in principle prevent $c$-dependent terms at the quantum level. This again would spoil the consistency of this procedure for determining the transformation law of $\phi^{*}$. Thus one has to find the full quantum Master Equation and check that it indeed is $c$-independent before one is sure of having a consistent formulation.

It still remains to be checked whether the additional term (104) also suffices to guarantee that the quantum mechanical Master Equation for $S^{B V}$ is independent of the ghosts $c$. This is indded the case, and we then finally have the complete quantum Master Equation. From the variation of the action we get:

$$
\delta S_{e x t}=\frac{\delta^{r} S^{B V}}{\delta \phi_{i}^{*}} \frac{\delta^{r} S^{B V}}{\delta \phi_{j}^{*}}(-1)^{\epsilon_{i} \epsilon_{j}+\epsilon_{i}} \phi_{k}^{*} \bar{\Gamma}_{i j}^{k}+\frac{\delta^{r} S^{B V}}{\delta \phi_{i}^{*}}\left[(-1)^{\epsilon_{i}} \frac{\delta^{r} S^{B V}}{\delta \phi^{B}} u_{i}^{B}-i \hbar(-1)^{\epsilon_{i}+\epsilon_{j}} \bar{\Gamma}_{j i}^{j}\right]
$$


The Jacobian contributes with:

$$
J=-i \hbar\left[\frac{\delta^{r}}{\delta \phi_{i}^{*}}\left[\frac{\delta^{r} S^{B V}}{\delta \phi^{B}} u_{i}^{B}\right]+\frac{\delta^{r}}{\delta \phi_{i}^{*}}\left[\frac{\delta^{r} S^{B V}}{\delta \phi_{j}^{*}}(-1)^{\epsilon_{i} \epsilon_{j}} \phi_{k}^{*} \bar{\Gamma}_{i j}^{k}\right]\right] .
$$

We get finally the following quantum Master Equation:

$$
\begin{aligned}
0=\delta S_{e x t}+ & J=\frac{\delta^{r} S^{B V}}{\delta \phi_{i}^{*}} \frac{\delta^{r} S^{B V}}{\delta \phi_{j}^{*}}(-1)^{\epsilon_{i} \epsilon_{j}+\epsilon_{i}} \phi *_{k} \bar{\Gamma}_{i j}^{k}+\frac{\delta^{r} S^{B V}}{\delta \phi_{i}^{*}}\left[(-1)^{\epsilon_{i}} \frac{\delta^{r} S^{B V}}{\delta \phi^{B}} u_{i}^{B}\right] \\
& -i \hbar\left[(-1)^{\left(\epsilon_{i}+1\right)\left(\epsilon_{B}+1\right)} \frac{\delta^{r}}{\delta \phi_{i}^{*}}\left(\frac{\delta^{r} S^{B V}}{\delta \phi^{B}}\right) u_{i}^{B}+(-1)^{\epsilon_{j}} \frac{\delta^{r}}{\delta \phi_{i}^{*}}\left(\frac{\delta^{r} S^{B V}}{\delta \phi_{j}^{*}}\right) \phi_{k}^{*} \bar{\Gamma}_{i j}^{k}\right] .
\end{aligned}
$$

When does the more general Master Equation (105) have non-trivial solutions? Let us first recapitulate some basic facts about the solutions to the conventional classical Master Equation

$$
\frac{1}{2}\left(S^{B V}, S^{B V}\right)=\frac{\delta^{r} S^{B V}}{\delta \phi^{A}} \frac{\delta^{l} S^{B V}}{\delta \phi_{A}^{*}}=0 .
$$

Since the most fundamental boundary condition is $S^{B V}\left[\phi, \phi^{*}=0\right]=S[\phi]$, where $S$ is the classical action, it is natural to assume that $S^{B V}$ will have an expansion in terms of antighosts $\phi^{*}$ :

$$
S^{B V}\left[\phi, \phi^{*}\right]=S[\phi]+\phi_{A}^{*} \mathcal{R}^{A}(\phi)+\ldots
$$

Inserting this into the classical Master Equation (113), and keeping only the first order in $\phi^{*}$, leads to the equation

$$
\left(\frac{\delta^{r} S}{\delta \phi^{A}}+\phi_{B}^{*} \frac{\delta^{r} \mathcal{R}^{B}}{\delta \phi^{A}}\right) \mathcal{R}^{A}=0 .
$$

This can only be satisfied if simultaneously

$$
\frac{\delta^{r} S}{\delta \phi^{A}} \mathcal{R}^{A}=0
$$

and

$$
\phi_{B}^{*} \frac{\delta^{r} \mathcal{R}^{B}}{\delta \phi^{A}} \mathcal{R}^{A}=0
$$

The first of these two equations says that the classical action must be invariant with respect to "internal" BRST transformations $\mathcal{R}$ :

$$
\delta \phi^{A}=\mathcal{R}^{A},
$$

while the second of these two equations expresses the condition that this symmetry be nilpotent:

$$
\delta^{2} \phi^{B}=\delta \mathcal{R}^{B}=\frac{\delta^{r} \mathcal{R}^{B}}{\delta \phi^{A}} \mathcal{R}^{A}=0
$$

To lowest order in an expansion in antighosts $\phi^{*}$, there is thus, with the boundary condition $S^{B V}[\phi, 0]=S[\phi]$ imposed, precisely a non-trivial solution to the classical Master Equation whenever the classical action is invariant under a nilpotent BRST symmetry.

Consider now the more general classical Master Equation (105). We again impose the condition $S^{B V}[\phi, 0]=S[\phi]$ (because otherwise we do not recover the correct Ward Identities from $S^{B V}$ ), and hence assume an expansion

$$
S^{B V}\left[\phi, \phi^{*}\right]=S[\phi]+\phi_{i}^{*} r^{i}(\phi)+\ldots
$$

\footnotetext{
${ }^{6} \mathrm{~A} \phi^{*}$-independent action $S^{B V}\left[\phi, \phi^{*}\right]=S[\phi]$ is of course always a solution.
} 
Retaining only terms up to one power of antighosts $\phi^{*}$ means that the classical Master Equation turns into

$$
-\left(\frac{\delta^{r} S}{\delta \phi^{A}}+\phi_{i}^{*} \frac{\delta^{r} r^{i}}{\delta \phi^{A}}\right) u_{j}^{A}(\phi) r^{j}(\phi)+\frac{1}{2}(-1)^{\epsilon_{j}\left(\epsilon_{i}+1\right)} r^{i}(\phi) r^{j}(\phi) \phi_{k}^{*} c_{i j}^{k}=0 .
$$

Also here this equation is actually equivalent to two separate requirements (since different powers of $\phi^{*}$ must cancel individually):

$$
\frac{\delta^{r} S}{\delta \phi^{A}} u_{i}^{A} r^{i}=0
$$

and

$$
-\phi_{i}^{*} \frac{\delta^{r} r^{i}}{\delta \phi^{A}} u_{j}^{A} r^{j}+\frac{1}{2}(-1)^{\epsilon_{j}\left(\epsilon_{i}+1\right)} r^{i} r^{j} \phi_{k}^{*} c_{i j}^{k}=0 .
$$

The first implies that the classical action must be invariant under a certain internal BRST symmetry,

$$
\delta \phi^{A}=u_{i}^{A} r^{i},
$$

but the second condition is not at first sight related to nilpotency of this BRST transformation. Consider, however, the condition $\delta^{2} \phi^{A}=0$ :

$$
\delta^{2} \phi^{A}=\delta\left[u_{i}^{A} r^{i}\right]=u_{i}^{A} \frac{\delta^{r} r^{i}}{\delta \phi^{B}} u_{j}^{B} r^{j}+(-1)^{\epsilon_{i}} \frac{\delta^{r} u_{i}^{A}}{\delta \phi^{B}} u_{j}^{B} r^{j} r^{i}=0
$$

Next, multiplying the identity (125) from the right by $(-1)^{\epsilon_{i}} r^{j} r^{i}$, and summing over $i$ and $j$ gives

$$
(-1)^{\epsilon_{i}} \frac{\delta^{r} u_{i}^{A}}{\delta \phi^{B}} u_{j}^{B} r^{j} r^{i}=-\frac{1}{2}(-1)^{\epsilon_{i}} u_{k}^{A} c_{i j}^{k} r^{j} r^{i}
$$

which means that nilpotency of the transformation (124) can be expressed as

$$
u_{i}^{A} \frac{\delta^{r} r^{i}}{\delta \phi^{B}} u_{j}^{B} r^{j}-\frac{1}{2}(-1)^{\epsilon_{i}} u_{k}^{A} c_{i j}^{k} r^{j} r^{i}=0 .
$$

It is now straightforward to verify that the condition $(123)$ is precisely equivalent to the requirement that the internal BRST symmetry $\delta \phi^{A}=u_{i}^{A} r^{i}$ is nilpotent. So as in the case of the conventional classical Master Equation, there is also here a direct link between having non-trivial solutions of the Master Equation to lowest order in a $\phi^{*}$-expansion, and having an internal nilpotent BRST symmetry of the action $S$. Furthermore, we see that the unusual commutator-term in the new Master Equation (105) is there to guarantee nilpotency of the internal BRST symmetry, once a solution to the equation has been found.

Only in the special case of an invertible $u_{i}^{A}$ does the argument also run in the inverse direction. There, given an internal nilpotent BRST symmetry, one can immediately write down the lowestorder solution to the Master Equation (since $r^{i}$ in that case is given explicitly in terms of the internal BRST symmetry). But in general, all one can infer is that if there is a non-trivial solution (120) to the new Master Equation (105) (and if one knows the set of transformations $g^{A}$ ) then the classical action will be BRST invariant with respect to the nilpotent transformations given above.

What, then, is the advantage of having a generalized Master Equation of the kind (105) available? In the conventional antibracket formalism the sole purpose of the Master Equation is to provide an extended action with the same physics as that of the original classical action (and, in its covariant generalizations, of the functional measure of these fields). But the extended action reduces to the original action if there are no internal symmetries to be fixed. So the real purpose 
of the standard antibracket formalism is to provide a systematic approach to the gauge fixing of internal symmetries. In the language of ref. [2] it is obvious why gauge fixing is so conveniently performed at the level of the extended action, rather than in the manner of the conventional Lagrangian BRST technique. This is because the nilpotent Schwinger-Dyson BRST symmetry is far more simple than that which can be encountered in the internal symmetries (including open algebras, reducible gauge symmetries etc.), and gauge fixing simply consists in the trivial addition of the (Schwinger-Dyson) BRST variation of a certain gauge fermion,

$$
\delta \Psi(\phi)=\frac{\delta^{r} \Psi}{\delta \phi^{A}} c^{a}
$$

to the extended action. Of course, when the classical action we are considering indeed has an internal BRST symmetry of the factorizable form (124), we can do the same kind of gauge fixing in the present formalism.

In performing that gauge fixing, it must be kept in mind that the BRST symmetry we use is the one on which the quantization itself is based, in this case the symmetry (70). It does not make any reference to possible internal BRST symmetries (instead, these surface automatically when we solve the Master Equation, and impose the proper boundary conditions). We thus add, for a certain gauge fermion $\Psi(\phi)$,

$$
\delta \Psi(\phi)=\frac{\delta^{r} \Psi}{\delta \phi^{A}} u_{i}^{A}(\phi) c^{i}
$$

to the extended action. After integrating out the ghosts $c^{i}$, the partition function can be written

$$
\mathcal{Z}=\int[d \phi]\left[d \phi^{*}\right] \delta\left(\phi_{i}^{*}-\frac{\delta^{r} \Psi}{\delta \phi^{A}} u_{i}^{A}\right) \exp \left[\frac{i}{\hbar} S^{B V}\right]
$$

where $S^{B V}$ is a solution to the Master Equation (105). This is essentially in the form of the Batalin-Vilkovisky prescription [1], (although, of course, the antighosts $\phi_{i}^{*}$ can no longer be viewed as "antifields" of the fields $\phi^{A}$ ) at least in the sense that the integration over the antighosts $\phi_{i}^{*}$ is trivial (due to the $\delta$-function arising from integrating over the ghosts $c^{i}$ ). The substitution

$$
\phi_{i}^{*} \rightarrow \frac{\delta^{r} \Psi}{\delta \phi^{A}} u_{i}^{A}
$$

in the solution to the Master Equation is thus what constitutes gauge fixing in this case. Indeed, in the action the result of such a substitution is

$$
S[\phi] \rightarrow S[\phi]+\frac{\delta^{r} \Psi}{\delta \phi^{A}} u_{i}^{A} r^{i}+\ldots,
$$

which, for a closed and irreducible internal algebra, would be the result of gauge fixing directly the internal symmetry $\delta \phi^{A}=u_{i}^{A} r^{i}$ (modulo quantum corrections). This is reassuring, because in the particular case of an invertible $u_{i}^{A}$ the quantization prescription based on the Master Equation (105) is equivalent to the one based on the conventional equation, and in this particular limit the two results should of course coincide.

\section{A New Bracket}

The more general Master Equation derived in subsection 4.2 hints at the existence of a new bracket structure which is more general than that of the usual antibracket. Since we in general will not have a one-to-one matching of fields $\phi^{A}$ and antighosts ("antifields") $\phi_{i}^{*}$, it is obvious that these 
fields cannot in general be canonical within the new bracket. However, just as the conventional antibracket can be viewed as a Grassmann-odd bracket based on a Heisenberg algebra between fields and antifields, a possible generalization of the antibracket can be based on a more general algebra. Indeed, as we shall now show, such a generalization is at the heart of the more general Master Equation formalism derived above.

\subsection{The more general "Quantum BRST Operator"}

To start, let us consider the by now simple problem of deriving the analogue of the "quantum BRST operator" associated with the more general BRST symmetry (51). We again wish to see how the BRST symmetry can be represented when the ghosts $c^{i}$ have been integrated out of the path integral. We use the identity

$$
\int[d c] F\left(c^{j}\right) e^{\frac{i}{\hbar} \phi_{i}^{*} c^{i}}=F\left(-i \hbar \frac{\delta^{l}}{\delta \phi_{j}^{*}}\right) \int[d c] e^{\frac{i}{\hbar} \phi_{i}^{*} c^{i}},
$$

and consider the BRST-variation of an arbitrary Green function $G$ depending only upon fields $\phi^{A}$ and antighosts $\phi_{i}^{*}$. For simplicity, consider the particular case when the functional measure is invariant under the transformation $\phi^{A} \rightarrow g^{A}\left(\phi^{\prime}, a\right)$. Using similar manipulations as in section 2.2 (most notably, a partial integration inside the path integral), we find that

$$
\delta G\left(\phi, \phi^{*}\right) \rightarrow \bar{\sigma} G\left(\phi, \phi^{*}\right)
$$

where

$$
\bar{\sigma} G \equiv\left[G, S^{B V}\right]-i \hbar \bar{\Delta} G
$$

Here $\bar{\Delta}$ is defined by

$$
\bar{\Delta} G \equiv(-1)^{\epsilon_{i}}\left[\frac{\delta^{r}}{\delta \phi^{A}} \frac{\delta^{r}}{\delta \phi_{i}^{*}} G\right] u_{i}^{A}+\frac{1}{2}(-1)^{\epsilon_{i}+1}\left[\frac{\delta^{r}}{\delta \phi_{j}^{*}} \frac{\delta^{r}}{\delta \phi_{i}^{*}} G\right] \phi_{k}^{*} c_{j i}^{k},
$$

and $[\cdot, \cdot]$ denotes a new Grassmann-odd bracket:

$$
[F, G] \equiv(-1)^{\epsilon_{i}\left(\epsilon_{A}+1\right)} \frac{\delta^{r} F}{\delta \phi_{i}^{*}} u_{i}^{A} \frac{\delta^{l} G}{\delta \phi^{A}}-\frac{\delta^{r} F}{\delta \phi^{A}} u_{i}^{A} \frac{\delta^{l} G}{\delta \phi_{i}^{*}}+\frac{\delta^{r} F}{\delta \phi_{i}^{*}} \phi_{k}^{*} c_{i j}^{k} \frac{\delta^{l} G}{\delta \phi_{j}^{*}} .
$$

\subsection{Properties of the New Bracket}

The bracket structure $[\cdot, \cdot]$ defined above is what enters naturally when one considers the BRST operator at the level where the ghosts $c^{i}$ have been integrated out, but their antighost partners $\phi_{i}^{*}$ have been kept. It is not obvious that such a bracket structure is of relevance beyond that stage. But the new bracket turns out to possess a number of useful properties that elevates it to a somewhat higher status. These properties are all shared with the conventional antibracket.

First, one easily verifies that the new bracket indeed is statistics-changing in the sense that $\epsilon([F, G])=\epsilon(F)+\epsilon(G)+1$. It satisfies an exchange relation of the kind

$$
[F, G]=(-1)^{\epsilon_{F} \epsilon_{G}+\epsilon_{F}+\epsilon_{G}}[G, F]
$$

and acts like a derivation with the rules

$$
[F, G H]=[F, G] H+(-1)^{\epsilon_{G}\left(\epsilon_{F}+1\right)} G[F, H]
$$




$$
[F G, H]=F[G, H]+(-1)^{\epsilon_{G}\left(\epsilon_{H}+1\right)}[F, H] G .
$$

It is straightforward, but rather tedious, to check that it also satisfies the super Jacobi identity

$$
(-1)^{\left(\epsilon_{F}+1\right)\left(\epsilon_{H}+1\right)}[F,[G, H]]+\text { cyclic perm. }=0 .
$$

(The ingredients needed to show this are the super Lie equations (40), and the Jacobi identity (45) for the structure coefficients $c_{j k}^{i}$ ).

Although all of these relations are shared by the conventional antibracket, the new bracket of course does not follow from the antibracket. On the contrary, the bracket $[\cdot, \cdot]$ is more general than the antibracket, to which it reduces in the trivial limit $u_{i}^{A}=\delta_{i}^{A}$ :

$$
[F, G]=(F, G) \quad \text { when } \quad u_{i}^{A}=\delta_{i}^{A} .
$$

Actually, the relation between the two brackets is slightly more general, since the bracket $[\cdot, \cdot]$ can be reduced to the usual antibracket $(\cdot, \cdot)$ through a redefinition of the antighosts $\phi_{i}^{*}$ whenever $u_{i}^{A}$ is invertible. This is, however, obviously a very special case as well. The relations (138)-(140) define what has been called a Gerstenhaber algebra [13].

Not surprisingly, it turns out that the classical Master Equation (105) can be expressed in terms of the more general bracket:

$$
\left[S^{B V}, S^{B V}\right]=0 .
$$

It is also interesting to consider the way the bracket acts on the fields $\phi^{A}$ and antighosts $\phi_{i}^{*}$. Clearly, there can be no canonical relations à la Poisson brackets since the number of fields $\phi^{A}$ in general is different from the number of antighosts $\phi_{i}^{*}$. Instead,

$$
\left[\phi^{A}, \phi_{i}^{*}\right]=u_{i}^{A},
$$

while within this bracket the antighosts satisfy a (super)Lie algebra:

$$
\left[\phi_{i}^{*}, \phi_{j}^{*}\right]=\phi_{k}^{*} c_{i j}^{k} .
$$

In addition, the bracket between two fields vanishes: $\left[\phi^{A}, \phi^{B}\right]=0$.

There are also relations between the $\bar{\Delta}$-operator and the new bracket. Consider the way $\bar{\Delta}$ acts on a product:

$$
\bar{\Delta}(F G)=F(\bar{\Delta} G)+(-1)^{\epsilon_{G}}(\bar{\Delta} F) G+(-1)^{\epsilon_{G}}[F, G] .
$$

As noticed by Witten [14], one can use this equation to define the new bracket $[\cdot, \cdot]$, given $\bar{\Delta}$. This approach is particularly useful in view of the fact that $\bar{\Delta}$ is nilpotent:

$$
\bar{\Delta}^{2}=0
$$

A general theorem [13] then assures that both the super Jacobi identity (160) and the exchange relation are satisfied automatically. Furthermore, using $\bar{\Delta}^{2}(F G)=0$, one finds

$$
\bar{\Delta}[F, G]=[F, \bar{\Delta} G]-(-1)^{\epsilon_{G}}[\bar{\Delta} F, G] .
$$

When the functional measure is invariant under $\phi^{A} \rightarrow g^{A}\left(\phi^{\prime}, a\right)$, we can also express the full quantum Master Equation entirely in terms of $\bar{\Delta}$ and the new bracket. From eq. (112) it follows that in this case,

$$
\frac{1}{2}\left[S^{B V}, S^{B V}\right]=i \hbar \bar{\Delta} S^{B V} .
$$


It is interesting to note that, just as in the case of the conventional antibracket, this equation can be compactly expressed as

$$
\bar{\Delta} e^{\frac{i}{\hbar} S^{B V}}=0 .
$$

A certain geometric interpretation [14, 15] probably underlies some of these observations. In the integration theory on the supermanifold spanned by the fields $\phi^{A}$ and the antighosts $\phi_{i}^{*}$, the operator $\bar{\Delta}$ should be viewed as a divergence operator associated with the invariant measure $\left[d \phi^{*}\right][d \phi] \rho(\phi)$. In the language of ref. [14], the quantum Master Equation (148) implies that $\exp \left[(i / \hbar) S^{B V}\right]$ can be considered as a "closed form", annihilated by the exterior derivative defined by $\bar{\Delta}$. Similarly, the BRST operator $\delta$, which in the formulation where the ghosts $c^{i}$ have been integrated out, equals $\bar{\sigma}$ of eq. (135), can conveniently be related to the $\bar{\Delta}$-operation. Consider a theory without gauge symmetries, for which the connection to the Ward identities is particularly simple. Once we let $\bar{\sigma}$ act on an object $\phi_{i}^{*} F[\phi]$, we get (see also ref. [7]):

$$
\bar{\sigma}\left[\phi_{i}^{*} F(\phi)\right]=\bar{\Delta}\left[\left(\frac{i}{\hbar}\right) \phi_{i}^{*} F(\phi) e^{\frac{i}{\hbar} S^{B V}}\right] .
$$

This means that the Schwinger-Dyson BRST Ward identities can be viewed as a generalized Stokes theorem on the supermanifold. It is curious that there exists an extension of the supermanifold (in which all antighost directions are doubled by keeping the ghosts $c^{i}$ ) where this same statement is just a reflection of an ordinary BRST Ward Identity. The reason for this is to be found in the fact that the BRST symmetry considered is precisely determined by the invariance properties of the path integral measure. An equivalent expression for the divergence operation in the formulation in which the ghosts $c^{i}$ are kept can presumably be obtained by considering the transformation properties of all fields $\phi^{A}, \phi_{i}^{*}, c^{i}$ under reparametrizations.

\section{Conclusions}

The aim of this paper has been to explore the more general framework in which the BatalinVilkovisky Lagrangian BRST formalism is situated. Once it is realized that this formalism can be derived from an underlying principle, that of ensuring correct Schwinger-Dyson equations in the path integral through the BRST symmetry [2], it becomes obvious how one can generalize it in various different directions. For example, the original Batalin-Vilkovisky construction [1] was concerned with theories whose functional measures were invariant under arbitrary local field shifts. The Schwinger-Dyson equations in those cases follow precisely from exploiting this shift invariance. If instead the functional measures are invariant under more general transformations - e.g., motion on a curved manifold - one can derive from first principles the analogue of the Batalin-Vilkovisky formalism for such cases by again promoting the corresponding symmetry transformation to a BRST symmetry. By following exactly the same procedure as in the flat case, we have shown how one arrives at a new Lagrangian BRST scheme, this time covariant with respect to transformations that leave the measure for the fields $\phi^{A}$ invariant. The result coincides with the recent generalization of the Batalin-Vilkovisky formalism that is inferred from general covariance arguments on the supermanifold spanned by fields $\phi^{A}$ and antifields $\phi_{A}^{*}$ alone [5]. Here, instead, we are by construction concerned only with covariance on the space of fields $\phi^{A}$, but this is of course just a special case. We emphasize that this covariant description of the Batalin-Vilkovisky formalism here is derived from first principles. It is only by keeping also the ghosts $c^{A}$ that we can uncover the underlying BRST principle behind this covariant generalization. It reads

$$
\delta \phi^{A}=u_{B}^{A}(\phi) c^{B}
$$




$$
\begin{aligned}
\delta c^{A} & =-\frac{1}{2}(-1)^{\epsilon_{B}} c_{B C}^{A} c^{C} c^{B} \\
\delta \phi_{A}^{*} & =(-1)^{\epsilon_{A}} \frac{\delta^{r} S}{\delta \phi^{B}} u_{A}^{B}(\phi)+i \hbar(-1)^{\epsilon_{A}+\epsilon_{B}} \bar{\Gamma}_{B A}^{B}+(-1)^{\epsilon_{A} \epsilon_{B}} \phi_{M}^{*} \bar{\Gamma}_{B A}^{M} c^{B},
\end{aligned}
$$

in the non-Abelian formulation. As expected, this makes explicit reference to both the action and the functional measure.

There is clearly no obstacle to considering also transformations that are $\phi^{*}$-dependent, which would lead to the formulation that is fully covariant on the supermanifold spanned by $\phi^{A}$ and $\phi_{A}^{*}$. But we see no physical principle that could motivate such a mixing of fields and antifields. From our point of view, the antifields $\phi_{A}^{*}$ are just particular antighosts (remnants of the articially introduced gauge symmetry associated with the given field redefinition), and not more special or important than the ghosts $c^{A}$. We might thus just as well consider transformations that involve these ghosts $c^{A}$ too.

As we have shown, one can go further, and consider elevating an arbitrary field transformation (which does not necessarily leave the measure invariant) into a BRST principle. The Ward identities of this BRST symmetry will then be the set of relations among Green functions that can be derived from the path integral by performing such a field redefinition. In general, this will correspond to subsets of the full set of Schwinger-Dyson equations. For this reason, it cannot in general be used as a principle on which to base the quantization procedure.

Even when the path integral measure is invariant under the given field transformation, this may not necessarily lead to the full set of Schwinger-Dyson equations. This is because the object $u_{i}^{A}(\phi)$ that enters into the corresponding BRST symmetry may not be invertible. When this is the case, one arrives at a bracket structure $[\cdot, \cdot]$ which is distinct from the conventional antibracket $(\cdot, \cdot)$. We have explored various properties of this new bracket in some detail, and found that it shares a number of features with the usual antibracket, to which it reduces when $u_{i}^{A}=\delta_{i}^{A}$.

The derivation of covariant Batalin-Vilkovisky quantization from the underlying SchwingerDyson BRST symmetry can also be generalized to the case of extended BRST symmetry [17, 18]. It would be most interesting to see how this how this compares with the recent covariant formulation of Batalin, Marnelius and Semikhatov [19, 20].

ACKnowledgement: The work of J.A. has been partially supported by Fondecyt 1950809, El Programa del Gobierno Español de Cooperación Científica con Iberoamérica and a collaboration CNRS-CONICYT.

\section{Appendices}

\section{A Actions on Lie groups}

When discussing the case of theories with non-trivial measures, we implicitly assumed that a natural choice of variables in the path integral would be given independently of the (super) Lie group of transformations that left the functional measure invariant. It often happens that the classical action itself is expressed directly in term of group elements, and that we wish to integrate over the left or right invariant measure on this group. It is therefore of interest to see how the Schwinger-Dyson

BRST symmetry, the associated Master Equation for the extended action $S_{\text {ext }}$, and the rest of the considerations above carry over to this case. 
First, how do we determine the most general Schwinger-Dyson equations? One way of answering this question is to find the appropriate generalization of "translations" on a given (super) group manifold. We shall outline a more general definition below, but let us first content ourselves with the same approach as in section 2.2 above. That is, we shall explore the identities that follow from using the fact that the functional measure on the group is chosen to be either left or right invariant (or both, but this is not needed). We shall for simplicity take the Lie group to be free of Grassmann-odd directions.

The fields $\phi^{A}$ are thus taken to be matrices $U(x)$, elements of the Lie group $G$. The notion of a Lie derivative $\nabla^{a}$ acting on group elements is useful at this stage. One can choose it to be

$$
\nabla^{a} \equiv i\left(U t^{a}\right)_{i j} \frac{\delta}{\delta U_{i j}}
$$

where $t^{a}$ are the generators of the group, with $\left[t^{a}, t^{b}\right]=i f_{c}^{a b} t^{c}$, and normalized to, say, $\operatorname{tr}\left(t^{a} t^{b}\right)=$ $\frac{1}{2} \delta^{a b}$. This Lie derivative acts much as an ordinary derivative, with, e.g., Taylor expansions of the form

$$
f\left(U e^{i \theta_{a} t^{a}}\right)=f(U)+\theta_{a} \nabla^{a} f(U)+\frac{1}{2} \theta_{a} \theta_{b} \nabla^{a} \nabla^{b} f(U)+\ldots
$$

Since Lie derivatives do not commute (but rather satisfy the Lie algebra itself), care is required when more than one derivative is involved.

Schwinger-Dyson equations for a theory of group elements $U(x)$, action $S[U]$, and partition function

$$
\mathcal{Z}=\int[d U] \exp \left[\frac{i}{\hbar} S[U]\right]
$$

take the form

$$
\left\langle\nabla^{a} F[U]+\left(\frac{i}{\hbar}\right) F[U] \nabla^{a} S[U]\right\rangle=0,
$$

for an arbitrary function $F[U]$. These equations are Ward identities of the equivalent theory based on [3]

$$
\mathcal{Z}=\int[d U]\left[d \phi^{*}\right][d c] \exp \left[\frac{i}{\hbar}\left(S[U]-\phi_{a}^{*} c^{a}\right)\right] .
$$

We view what is in the exponent as the extended action, and denote it by $S_{\text {ext }}$. The BRST symmetry under which both the action and the right-invariant measure remains invariant reads

$$
\begin{aligned}
\delta U(x) & =i U(x) t_{a} c^{a} \\
\delta c^{a}(x) & =0 \\
\delta \phi_{a}^{*} & =-\nabla_{a} S_{e x t}
\end{aligned}
$$

where in the last line we have used the decoupling of the ghost fields from the classical action to rewrite the symmetry in terms of $S_{\text {ext }}$. The Ward Identities of this symmetry are Schwinger-Dyson equations.

The above BRST symmetry suffers from not being nilpotent, even when just acting on the group elements $U$. We can remedy this by hand if we change the transformation law for the ghosts $c^{a}$. This will not affect the Ward Identities $0=\left\langle\delta\left\{\phi_{a}^{*} F[U]\right\rangle\right.$, which hence still provide correct Schwinger-Dyson equations. The required modification is

$$
\delta c^{a}=\frac{1}{2} c_{b c}^{a} c^{b} c^{c}
$$


Now $\delta^{2} U=0$, as required, but the action in (156) is no longer invariant under the BRST symmetry. We can again correct for this by hand if we modify the transformation law for $\phi^{*}$ as well:

$$
\delta \phi_{a}^{*}=-\nabla_{a} S_{e x t}+\frac{1}{2} \phi_{b}^{*} c_{c a}^{b} c^{c} .
$$

On the surface this would seem to change the above Ward Identity. But when we perform the ghostantighost integrations, the extra term is seen to contribute a term proportional to $c_{a b}^{a}$. This term hence vanishes whenever the Lie group is semi-simple (a requirement we had to impose anyway, because otherwise the integration measure for the ghosts $c^{a}$ would not be invariant under the modified transformation law proposed above).

The above Schwinger-Dyson BRST symmetry implies that the extended action satisfies a certain Master Equation. For the non-nilpotent version (157) it reads

$$
\frac{\delta^{r} S_{\text {ext }}}{\delta \phi_{a}^{*}} \nabla_{a} S_{\text {ext }}=c^{a} \nabla_{a} S_{\text {ext }} .
$$

It is clear that once a solution has been found, one can replace the ghosts $c^{a}$ by $c^{a}+\alpha \mathcal{R}^{a}$, where $\alpha$ is an arbitrary constant, and $\mathcal{R}^{a}$ is annihilated by $\nabla_{a} S_{\text {ext }}$ :

$$
\mathcal{R}^{a} \nabla_{a} S_{\text {ext }}=0
$$

Gauge symmetries involving solely fields that are elements of a compact Lie group $G$ ordinarily need not be gauge fixed. But the Schwinger-Dyson BRST symmetry (157) is independent of possible internal gauge symmetries, and is valid in general - as is the Master Equation (160) for such theories. Since normally no other ghost fields will be present, the solution (156) suffices. However, one can conceive of situations where gauge fixing is convenient, and where extra ghost fields then have to be introduced, even in this context. These extra fields will not be elements of the group $G$, and one therefore has to specify additionally their BRST transformation law, on top of the list given in eq. (157). Once specified, one can immediately write down the corresponding (quantum) Master Equation by demanding that the BRST variation of the action is cancelled by that of the measure - as was done in section 3 .

As this example has shown, the Master Equation can take quite different forms depending on the field theory context. The antibracket does not enter at all in the present case, and the Master Equation involves fields $U(x) \in G$ that are on a quite different footing from their natural partners in Batalin-Vilkovisky quantization, the antighosts $\phi^{*}$ (which belong to the algebra of the group). Still, this is the direct group theory analogue of the Batalin-Vilkovisky formalism.

\section{B A more general Setting}

Let $\mathcal{M}$ be a manifold and $G$ a group of transformations of $\mathcal{M}$. For convenience, let us here restrict ourselves to bosonic manifolds. We will say that $G$ acts transitively on $\mathcal{M}$ if given $x, y$ in $\mathcal{M}$ there exists an element $g$ in $G$ such that

$$
y=g * x
$$

Actually, our future analysis will deal only with local properties of $\mathcal{M}$. So we will need a less restrictive property of $G$, local transitivity. That is, $\mathrm{G}$ acts transitivily on every neighborhood of a given point of $\mathcal{M}$.

\footnotetext{
${ }^{7}$ For some recent examples, see, e.g., ref. [21].
} 
Let $x_{0}$ be a point of $\mathcal{M}$. The little group of $x_{0}\left(H_{x_{0}}\right)$ is the subgroup of elements in $G$ that do not change $x_{0}$. We will refer to it intuitively as "rotations". i.e.:

$$
x_{0}=h * x_{0} \quad, \quad h \in H_{x_{0}}
$$

It is easy to show that if $G$ acts transitively in a certain submanifold of $\mathcal{M}$, then $H$ is independent of the basis point $x_{0}$ in that region. From now on we will assume this to be the case, and will hence not write explicitly the subindex of $H$.

Let $\mathcal{M}_{0}$ be a submanifold of $\mathcal{M}$ (the neighborhood of a point $x_{0}$ for instance) and $G$ a group that acts transitively on $\mathcal{M}_{0}$. Then it follows that $\mathcal{M}_{0}$ is isomorphic to $G / H$, where $H$ is the little group of $\mathcal{M}_{0}$. We will refer to the elements of $G / H$ as "translations". $G / H$ is called a symmetric space in the literature. Since we only need the local property, we will refer to it as locally symmetric space.

From now on we will study path integral quantization on locally symmetric spaces. According to the last paragraph this covers a large class of manifolds.

We will obtain Schwinger-Dyson equations only from "translations". This is the most general set of identities in cases of interest because normally the action $S$ is invariant under "rotations". If this were not the case, then the most general invariance of the measure (both "translations" and "rotations") should be explored. Then there will of course be more antifields than fields.

Our object of interest is the following functional integral:

$$
\int[d x] \rho(x) e^{-S[x]}
$$

We will assume that the group of symmetries $G$ of the functional measure acts transitively in a neighborhood of each point of $\mathcal{M}$. So we can choose as local coordinates the elements of $G / H, x$. Moreover we can choose the integration measure to be the Haar measure of $G$.

We apply now the collective coordinates method. Let $a$ be an element of $G / H$. Then we consider:

$$
\int[d a] \rho[a] \int[d x] \rho[x] e^{-S\left[a^{-1} * x\right]}
$$

The new action is invariant under left multiplication,

$$
\begin{aligned}
\tilde{S}[x, a] & =S\left[a^{-1} * x\right] \\
a^{\prime} & =b * a \\
x^{\prime} & =b * x
\end{aligned}
$$

In order to use the BRST method we need the infinitesimal transformation. Let us choose variables such that $x=0$ corresponds to the identity of $G . \phi(a, x)$ will be the (real) parameter corresponding to the element $a * x$. This corresponds to the left multiplication rule. Then the infinitesinal transformations are:

$$
\begin{aligned}
\delta a_{\alpha} & =\Theta_{\alpha \beta}(a) b^{\beta} \\
\delta x_{\alpha} & =\Theta_{\alpha \beta}(x) b^{\beta} \\
\Theta_{\alpha \beta} & =\left.\frac{\partial \phi(b, a)_{\alpha}}{\partial b^{\beta}}\right|_{b=0}
\end{aligned}
$$

The corresponding BRST transformation is:

$$
\delta a_{\alpha}=\Theta_{\alpha \beta}(a) c^{\beta}
$$




$$
\begin{aligned}
\delta x_{\alpha} & =\Theta_{\alpha \beta}(x) c^{\beta} \\
\delta c^{\beta} & =\frac{1}{2} c_{\epsilon \gamma}^{\beta} c^{\epsilon} c^{\gamma} \\
\delta \bar{c}_{\beta} & =i b_{\beta} \\
\delta b_{\beta} & =0 .
\end{aligned}
$$

Here $c_{\epsilon \gamma}^{\beta}$ are the structure constants of the Lie group. It is easy to check that the BRST generator correponding to this set of transformations is nilpotent.

Now we fix for convenience the gauge $a=0$. The gauge fixed action is:

$$
\bar{S}=S\left[a^{-1} * x\right]-i b a-\bar{c}_{\alpha} \Theta_{\alpha \beta}(a) c^{\beta}
$$

Integration over $b$ and $a$ gives:

$$
S_{\text {ext }}=S(x)-\bar{c}_{\alpha} c^{\alpha}
$$

which is invariant under

$$
\begin{aligned}
\delta x_{\alpha} & =\Theta_{\alpha \beta}(x) c^{\beta} \\
\delta c^{\beta} & =\frac{1}{2} c_{\epsilon \gamma}^{\beta} c^{\epsilon} c^{\gamma} \\
\delta \bar{c}_{\beta} & =-\frac{\partial S}{\partial x^{\sigma}} \Theta_{\sigma \beta}(x)+\left.\bar{c}_{\alpha} \frac{\partial \Theta_{\alpha \gamma}}{\partial a^{\beta}}\right|_{a=0} c^{\gamma} .
\end{aligned}
$$

The generalization to supermanifolds is straightforward. 


\section{References}

[1] I.A. Batalin and G.A. Vilkovisky, Phys. Lett. 102B (1981) 27; Phys. Rev. D28 (1983) 2567 [E: D30 (1984) 508]; Nucl. Phys. B234 (1984) 106; J. Math. Phys. 26 (1985) 172.

[2] J. Alfaro and P.H. Damgaard, Nucl. Phys. B404 (1993) 751.

[3] J. Alfaro and P.H. Damgaard, Phys. Lett. B222 (1989) 425.

J. Alfaro, P.H. Damgaard, J. Latorre and D. Montano, Phys. Lett. B233 (1989) 153.

[4] M. Henneaux, Nucl. Phys. B (Proc. Suppl.) 18A (1990) 47.

[5] O.M. Khudaverdian, J. Math. Phys. 32 (1991) 1934.

O.M. Khudaverdian and A.P. Nersessian, Mod. Phys. Lett. A8 (1993) 2377.

A. Schwarz, Commun. Math. Phys. 155 (1993) 249; 158 (1993) 373.

I.A. Batalin and I.V. Tyutin, Int. J. Mod. Phys. A8 (1993) 2333; Mod. Phys. Lett. A8 (1993) 3673 .

H. Hata and B. Zwiebach, Ann. Phys. (NY) 322 (1994) 131.

[6] J. Alfaro and P.H. Damgaard, Phys. Lett. B334 (1994) 369.

[7] M. Henneaux and C. Teitelboim: Quantization of Gauge Systems, Princeton University Press (New Jersey) 1992.

[8] F.W. Hehl, P. von der Heyde and G.D. Kerlick, Rev. Mod. Phys. 48 (1976) 393.

[9] A. Saa, preprint hep-th/9307095.

[10] J. Alfaro and P.H. Damgaard, Ann. Phys. (NY) 202 (1990) 398.

[11] A. Hosoya and K. Kikkawa, Nucl. Phys. B101 (1975) 271.

[12] J. Alfaro and P.H. Damgaard, Ann. Phys. (NY) 220 (1992) 188.

[13] M. Penkava and A. Schwarz, UC Davis preprint (1992), hep-th/9212072.

[14] E. Witten, Mod. Phys. Lett. A5 (1990) 487.

[15] M. Henneaux, Phys. Lett. B282 (1992) 372.

[16] M. Hamermesh: Group Theory and its Application to Physical Problems, Pergamon Press (London) 1964.

I.A. Batalin, J. Math. Phys. 22 (1981) 1837.

[17] I.A. Batalin, Lavrov and Tyutin, J. Math. Phys. 31 (1990) 1487; ibid. 32 (1990) 532; ibid. 32 (1990) 2513.

[18] P.H. Damgaard and F. de Jonghe, Phys. Lett. B305 (1993) 59.

[19] I.A. Batalin, R. Marnelius and A. Semikhatov, hep-th/9502031.

[20] A. Nersessian and P.H. Damgaard, hep-th/9505009.

[21] P.H. Damgaard and R. Sollacher, Phys. Lett. B322 (1994) 131; Nucl. Phys. 433 (1995) 671. 